
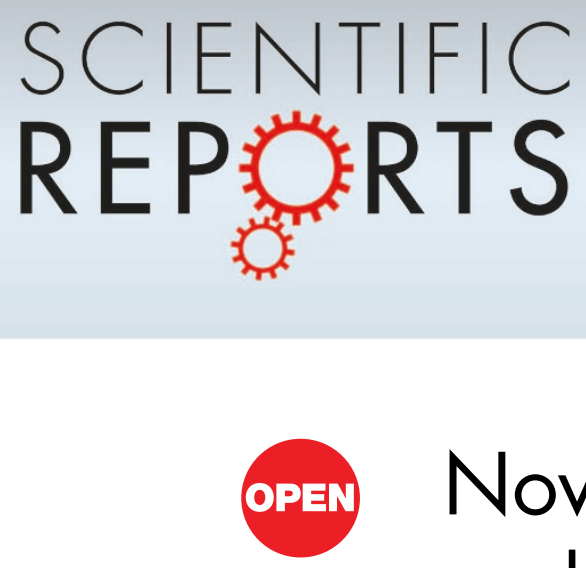

\title{
Novel n-3 Immunoresolvents: Structures and Actions
}

\author{
SUBJECT AREAS: \\ Jesmond Dalli, Romain A. Colas \& Charles N. Serhan
}

EXPERIMENTAL MODELS

OF DISEASE

ACUTE INFLAMMATION

INNATE IMMUNITY

CHRONIC INFLAMMATION

Received

5 March 2013

Accepted

13 May 2013

Published

5 June 2013

Correspondence and requests for materials should be addressed to

C.N.S. (cnserhan@ zeus.bwh.harvard. edu)

Center for Experimental Therapeutics and Reperfusion Injury, Department of Anesthesiology, Perioperative and Pain Medicine, Harvard Institutes of Medicine, Brigham and Women's Hospital and Harvard Medical School, Boston, Massachusetts. 02115.

Resolution of inflammation is now held to be an active process where autacoids promote homeostasis. Using functional-metabololipidomics and in vivo systems, herein we report that endogenous n-3

docosapentaenoic (DPA) acid is converted during inflammation-resolution in mice and by human leukocytes to novel $n-3$ products congenerous to $D$-series resolvins (Rv), protectins (PD) and maresins $(\mathrm{MaR})$, termed specialized pro-resolving mediators (SPM). The new n-3 DPA structures include 7,8,17-trihydroxy-9,11,13,15E,19Z-docosapentaenoic acid (RvD1 $\left.1_{n-3} \mathrm{DPA}\right)$, 7,14-dihydroxy-8,10,12,16Z,19Z-docosapentaenoic acid (MaR1 $1_{n-3}$ DPA $)$ and related bioactive products. Each n-3 DPA-SPM displayed protective actions from second organ injury and reduced systemic inflammation in ischemia-reperfusion. The n-3 DPA-SPM, including RvD1 $1_{n-3}$ DPA and MaR $1_{n-3}$ DPA each exerted potent leukocyte directed actions in vivo. With human leukocytes each n-3 DPA-SPM reduced neutrophil chemotaxis, adhesion and enhanced macrophage phagocytosis. Together, these findings demonstrate that n-3 DPA is converted to novel immunoresolvents with actions comparable to resolvins and are likely produced in humans when n-3 DPA is elevated.

. he resolution of acute inflammation is an active process temporally orchestrated by local-acting mediators that limit further neutrophil recruitment to sites of inflammation ${ }^{1-4}$. During the onset of inflammation, chemical mediators, including the arachidonic acid-derived eicosanoids (e.g. leukotriene (LT) $\mathrm{B}_{4}$ and prostaglandin $(\mathrm{PG}) \mathrm{E}_{2}$ ), mount initiation and propagation of inflammation ${ }^{5,6}$, actions that are actively counter-regulated and orchestrated by pro-resolution agonists ${ }^{1,2,4}$. These pro-resolving autacoids also stimulate the clearance of debris, apoptotic cells and bacteria, promoting homeostasis ${ }^{1,2,7}$.

One of the key steps during resolution of inflammation is an increase in local vascular permeability leading to edema $^{6,8}$ and the transport of n-3 essential fatty acids (EFA) from blood to the site of inflammation ${ }^{9}$. n-3 EFA are linked with protective actions in a number of inflammatory conditions including rheumatoid arthritis ${ }^{10}$, neurological disorders ${ }^{11}$ and cardiovascular disease ${ }^{12}$. At the site of inflammation, n-3 EFA are converted to novel potent mediators by exudate leukocytes that promote inflammation-resolution ${ }^{1,2,13}$.

The E-series resolvins, e.g. resolvin (Rv) E1, are produced from 5Z,8Z,11Z,14Z,17Z-eicosapentaenoic acid $(\mathrm{EPA})^{14}$. The D-series resolvins, which include Resolvin D1 (7S, 8R, 17S-trihydroxy-4Z, 9E, 11E, 13Z, 15E, 19Zdocosahexaenoic acid; RvD1) and Resolvin D2 (7S, 16R, 17S-trihydroxy-4Z, 8E, 10Z, 12E, 14E, 19Z-docosahexaenoic acid; RvD2), the protectins ${ }^{15}$ and maresins ${ }^{16}$ are biosynthesized from 4Z,7Z,10Z,13Z,16Z,19Z-docosahexaenoic acid (DHA). These potent autacoids were initially identified using temporal lipidomics with self-resolving exudates $^{14,15}$ and are now appreciated for their ability to stereo-selectively promote inflammation-resolution by tempering leukocyte responses ${ }^{16,17,13}$. The resolvins, protectins and maresins are coined specialized pro-resolving mediators (SPM) that by definition limit further neutrophil recruitment to the site of inflammation and promote macrophage clearance of debris, apoptotic cells and bacteria ${ }^{1,2,7}$. In addition, the SPM exert potent actions in promoting wound repair and tissue regeneration as well as dampening inflammatory pain ${ }^{16,18}$. Targeted lipid mediator metabololipidomics of tissues obtained from a number of species ranging from primordial organisms such as planaria ${ }^{16}$ to humans ${ }^{19,20}$ indicates that SPM production, including RvD1, RvE1 $1^{19,20}$ and maresin $1^{2,14}$, is evolutionarily conserved.

In mammals, alpha-linolenic acid (9Z, 12Z, 15Z-octadecatrienoic acid; ALA) is converted via elongation and desaturation to EPA and subsequently to DHA. An intermediate in the conversion of EPA to DHA is n-3 docosapentaenoic acid (7Z,10Z,13Z,16Z,19Z-docosapentaenoic acid; n-3 DPA) ${ }^{10,12,21}$. n-3 DPA carries 22 carbons and contains five double bonds, with the first double bond being found on carbon 7 . The structural differences of n-3 DPA from EPA, DHA and n-6 docosapentaenoic acid (4Z, 7Z, 10Z, 13Z, 16Z,-docosapentaenoic acid; n-6 DPA), a biochemically distinct form of DPA where the first double bond is found on carbon 4, are 
thought to confer unique biophysical properties that are of functional relevance, for example in neuronal systems ${ }^{22}$. In humans, genomewide association studies demonstrate that elevation in circulating levels of n-3 DPA and a concomitant decrease in DHA levels are associated with single nucleotide polymorphisms in the gene encoding for the fatty acid elongase $2(E L O V L 2)^{21}$. n-3 DPA is present in a number of mammalian tissues including plasma, brain, retina and heart at levels comparable to those of EPA and DHA ${ }^{23}$. Since circulating levels of n-3 DPA in human cohorts of European, African, Hispanic and Chinese ancestry with mutations in the ELOVL2 gene are elevated ${ }^{21}$, we addressed herein whether n-3 DPA is a precursor to novel bioactive molecules. We report that endogenous n-3 DPA is converted to novel bioactive products, determined their structures and established their anti-inflammatory and pro-resolving actions.

\section{Results}

n-3 docosapentaenoic acid products exert potent anti-inflammatory and tissue-protective actions in vivo. Since even minor changes in the structural properties of EFA are of functional significance ${ }^{22}$, we first investigated whether n-3 DPA, products that contain one fewer double bond than those derived from DHA (Fig. 1a), produced by exudate leukocytes exerted protective actions during acute inflammation. For this purpose, we employed a model of surgery-induced second organ injury, the murine hind limb ischemia reperfusion model (Fig. 1b) ${ }^{9}$. Administration of an isolated mixture obtained via solid-phase extraction (see Methods) of the n-3 DPA products $10 \mathrm{~min}$ prior to onset of reperfusion led to protection from secondary organ injury as evidenced by reduction in lung tissue damage (Fig. 1c) and decrease in the number of infiltrated leukocytes into the lungs $(\sim 45 \%, p<0.05)$. These actions were comparable to protection afforded by the DHA-derived pro-resolving mediator RvD1 (Fig. 1c, d).

Assessment of whole blood neutrophil-platelet aggregates in these mice, a marker of systemic inflammation ${ }^{24}$, following administration of n-3 DPA products or RvD1 gave a significant reduction (55-65\%) in the levels of platelet-leukocyte aggregates found $2 \mathrm{~h}$ post reperfusion (Fig. 1e).

Using targeted lipid mediator metabololipidomics, we next assessed whether these n-3 DPA products also regulated proinflammatory eicosanoid biosynthesis following ischemia reperfusion. Administration of these products led to a significant reduction in plasma prostanoid levels including $\mathrm{PGE}_{2}(\sim 75 \%)$ and thromboxane $\mathrm{B}_{2}\left(\mathrm{TxB}_{2}\right)(\sim 80 \%$; Fig. $1 \mathrm{~F})$. Here we also found a significant reduction in plasma $\mathrm{LTB}_{4}(\sim 60 \%)$ levels along with a decrease in levels of its double di-oxygenation isomer 5S,12S-diHETE ( $\sim 75 \%$; Fig. 1g). Of note the n-3 DPA products displayed equal or higher potency at regulating plasma eicosanoid levels when compared to RvD1 (Fig. 1f, g). These results demonstrate that n-3 DPA products possess potent anti-inflammatory and tissue-protective actions, regulating leukocyte recruitment, pro-inflammatory mediator biosynthesis and systemic inflammation.

Targeted metabololipidomics of plasma following ischemia reperfusion injury. Having found that n-3 DPA products display potent actions during ischemia reperfusion, we next investigated the role of endogenous n-3 DPA derived in the control of acute inflammation. First we assessed the plasma levels of unesterified arachidonic acid (AA), eicosapentaenoic acid (EPA), n-3 docosapentaenoic acid (DPA) and docosahexaenoic acid (DHA) in mice that were not subjected to an inflammatory stimulus. Plasma AA levels were $573.0 \mathrm{ng} / \mathrm{ml}$, EPA levels were $116.2 \mathrm{ng} / \mathrm{ml}, \mathrm{n}-3$ DPA levels were $66.3 \mathrm{ng} / \mathrm{ml}$ and DHA levels were $146.1 \mathrm{ng} / \mathrm{ml}$. Following ischemia reperfusion injury, circulating values for all 4 PUFA were elevated, with levels for $n-3$ DPA increasing $\sim 12$ times to those found in uninjured mice (Fig. 2a).
We next sought evidence for the conversion of n-3 DPA to bioactive mediators during acute inflammation. Using lipid mediator metabololipidomics and monitoring the precursor ion $\mathrm{m} / \mathrm{z} 345$ in $\mathrm{Q} 1$ and the product ion $\mathrm{m} / \mathrm{z} 327$ in Q3, we found three major products eluting in LC-peaks (Fig. 2b). Analysis of the tandem mass spectra (MS-MS) for the products under each peak demonstrated that peak $I$, at retention time $\left(\mathrm{R}_{\mathrm{T}}\right)=17.1 \mathrm{~min}$, corresponded to 17 HDPA (Supplementary Fig. 1a), the peak at $\mathrm{R}_{\mathrm{T}}=17.3$ min (peak II) to 14-HDPA (Supplementary Fig. 1b) and peak $I I I$ at $\mathrm{R}_{\mathrm{T}}=17.6 \mathrm{~min}$ corresponded to 7-HDPA (Supplementary Fig. 1c). Quantification of these novel products by multiple reaction monitoring (MRM) in the plasma of uninjured mice and mice subjected to ischemia reperfusion demonstrated that all three products were elevated following ischemia reperfusion injury (Fig. 1c). These results demonstrate that during acute inflammation, systemic n-3 DPA levels are elevated, and this n-3 EFA is converted to novel oxygenated products.

Chiral metabololipidomics of endogenous n-3 DPA products. In order to elucidate the biosynthetic origins of these novel n-3 DPAderived products we designed chiral metabololipidomic profiling of the n-3 DPA products generated in vivo. Reverse-phase chiral LCMS-MS metabololipidomics of plasma samples obtained from mice subjected to ischemia reperfusion injury achieved baseline separation of $7 R / S$-HDPA, 14R/S-HDPA and 17R/S-HDPA (Supplementary Fig. 2). Quantification of the two isomers for each of the products identified demonstrated that the $R$ to $S$ ratio for all of the monohydroxy products identified was $\sim 20 \%: \sim 80 \%$. These results indicated that the conversion of n-3 DPA to these novel products is enzymatically regulated, since mammalian lipoxygenases are known to insert molecular oxygen in predominantly the $S$ configuration ${ }^{5}$.

Targeted metabololipidomics during onset and resolution of acute inflammation uncovers novel $n-3$ docosapentaenoic acid products. Having found that n-3 DPA is converted in vivo to yield monohydroxy acids that predominantly possess the $S$ chirality, we next investigated whether these n-3 DPA monohydroxy products were precursors and/or pathway markers for the biosynthesis of bioactive mediators. To this end, we employed targeted LM metabololipidomics with plasma from mice subjected to ischemia reperfusion injury. Multiple reaction monitoring of $\mathrm{m} / \mathrm{z} 377$ in Q1 and $\mathrm{m} / \mathrm{z} 143$ in Q3 yielded two peaks, the first at $\mathrm{R}_{\mathrm{T}}=11.6 \mathrm{~min}$ and the second at $\mathrm{R}_{\mathrm{T}}=12.1 \mathrm{~min}$ (Fig. 3a). Inspection of the MS-MS spectrum for the product eluting in peak at $R_{T}=11.6 \mathrm{~min}$ demonstrated that this material corresponded to RvD2 $2_{n-3}$ DPA with the following characteristic ions assigned: $\mathrm{m} / \mathrm{z} 307, \mathrm{~m} / \mathrm{z} 279, \mathrm{~m} / \mathrm{z} 249$, $\mathrm{m} / \mathrm{z}$ 233, and $\mathrm{m} / \mathrm{z} 143$ (c.f. Supplementary Fig. 3a, b); while assessment of the MS-MS spectrum for the products at $\mathrm{R}_{\mathrm{T}}=$ 12.1 min demonstrated that this material corresponded to $\mathrm{RvD} 1_{\mathrm{n}-3}$ DPA (Fig. 3b). Multiple reaction monitoring of $\mathrm{m} / \mathrm{z} 361$ in $\mathrm{Q} 1$ and $\mathrm{m} / \mathrm{z}$ 263 in Q3 yielded three peaks, one at $\mathrm{R}_{\mathrm{T}}=13.6 \mathrm{~min}$, the second peak with $\mathrm{R}_{\mathrm{T}}=13.7 \mathrm{~min}$ and the third peak $\mathrm{R}_{\mathrm{T}}=14.4 \mathrm{~min}$ (Fig. $3 \mathrm{a}$ ). Assessment of MS-MS spectra for the product with $\mathrm{R}_{\mathrm{T}}=13.6 \mathrm{~min}$ gave characteristic fragmentation corresponding to $\mathrm{RvD} 5_{\mathrm{n}-3}$ DPA (Fig. 3c). MS-MS fragmentation for the material at $R_{T}=13.7 \mathrm{~min}$ demonstrated characteristic fragmentation corresponding to $\mathrm{PD} 1_{\mathrm{n}-3}$ DPA (Fig. 3d). The peak eluting at $\mathrm{R}_{\mathrm{T}}=14.4 \mathrm{~min}$ was identified as $\mathrm{PD} 2{ }_{\mathrm{n}-3}$ DPA with the following characteristic ions assigned: $\mathrm{m} / \mathrm{z} 233$, $\mathrm{m} / \mathrm{z} 247, \mathrm{~m} / \mathrm{z} 189$ (c.f. Supplementary Fig. 4c, d). These findings demonstrate that n-3 DPA is converted to novel products that are cognate to pro-resolving mediators from DHA; therefore, we employed the nomenclature from the DHA bioactive metabolome to describe each of these new structures.

Since vascular leakage during inflammation supplies the site of inflammation with PUFA $^{9}$, we next investigated tissue levels of n-3 DPA products during the onset and resolution of inflammation. For this purpose, we applied a self-limited model of inflammation where, following the administration of a pro-inflammatory stimulus into the 
a

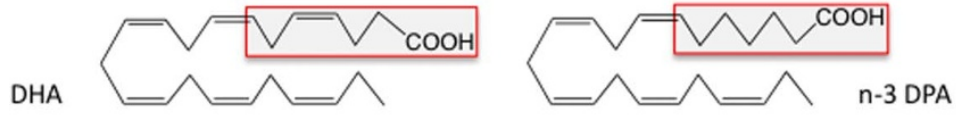

b

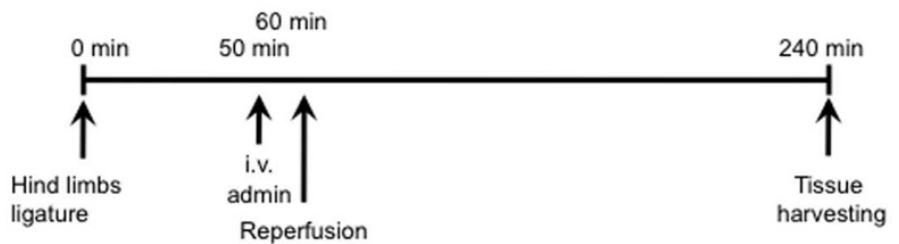

C

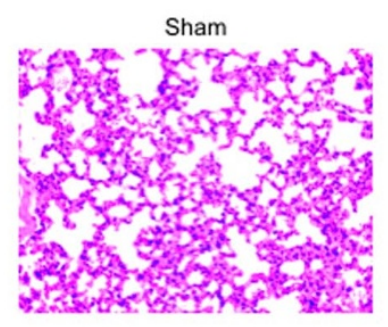

d

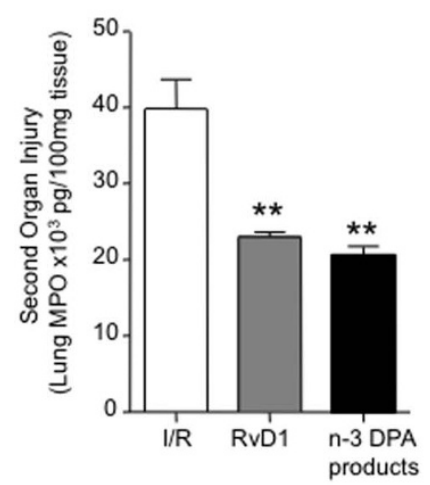

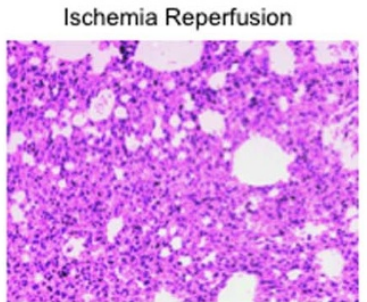

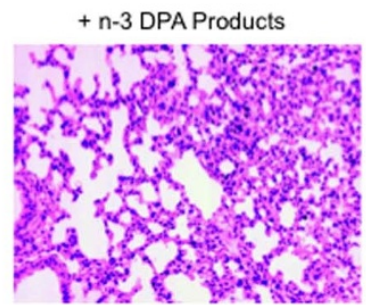

e

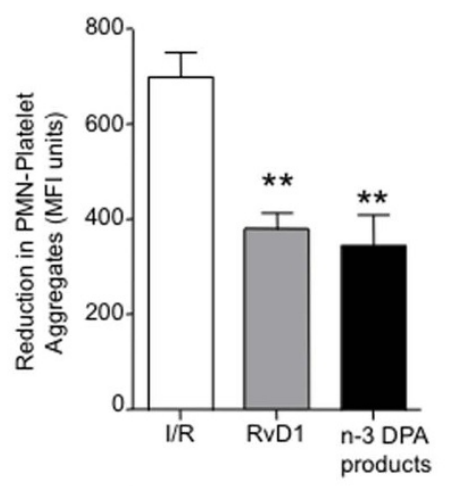

f

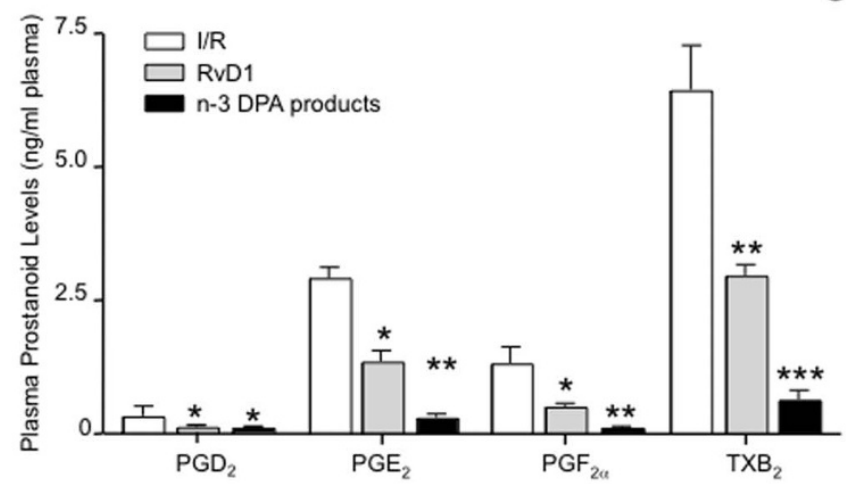

g

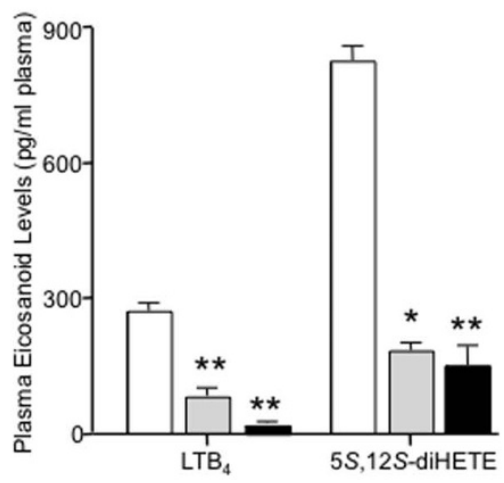

Figure $1 \mid$ n-3 DPA-derived products display potent anti-inflammatory and tissue protective actions in vivo that are comparable to RvD1.

(a) Structures of DHA and n-3 DPA. (b) Ischemia was induced by applying tourniquets to the hind limb of 6-8-week-old male FvB mice. After 1 h, tourniquets were removed and reperfusion ensued for $3 \mathrm{~h}$. $10 \mathrm{~min}$ prior to reperfusion, vehicle (saline containing $0.1 \% \mathrm{EtOH}$ ), RvD1 (500 ng) or a mixture of n-3 DPA-derived products (see Methods for details) were administered intravenously. At the end of reperfusion, lungs were collected; (c) tissue histology by H\&E staining (x200) and (d) MPO levels were assessed. (e) Blood was collected, incubated with rat anti-mouse Ly6G and rat antimouse CD41 antibodies and neutrophil leukocyte aggregates were assessed by flow cytometry. (f) Plasma prostanoid and (g) leukotriene levels were assessed by lipid mediator metabololipidomics. Results $\mathrm{c}$ are representative $\mathrm{n}=4$. Results $\mathrm{d}-\mathrm{e}$ are mean $\pm \mathrm{SEM}$. $\mathrm{n}=4$. ${ }^{*} P<0.05,{ }^{*} P<0.01$ vs. vehicle mice.

mouse peritoneum, there is a rapid recruitment of neutrophils into the site that peaks at around $6 \mathrm{~h}\left(\mathrm{~T}_{\max } ; \mathrm{Fig} .4 \mathrm{a}\right)$. This is followed by a decline in neutrophil numbers over the next $18 \mathrm{~h}$. The time difference between $\mathrm{T}_{\max }$ and the point where neutrophil numbers reach $50 \%$ of maximum $\left(\mathrm{T}_{50}\right)$ is defined as the resolution interval $\left(\mathrm{R}_{\mathrm{i}}\right)^{25}$. Using LM metabololipidomics, we profiled the levels of $\mathrm{LTB}_{4}$ and
$\mathrm{PGE}_{2}$ that were rapidly produced during the initiation phase of the inflammatory response concomitant with neutrophil infiltration into the tissue (Fig. 4b). Maximal $\mathrm{LTB}_{4}$ levels coincided with peak neutrophil infiltration, whereby $\mathrm{LTB}_{4}$ levels rapidly subsided over the next $8 \mathrm{~h}$. $\mathrm{PGE}_{2}$ levels were also elevated early in the initiation phase of the response, with levels for this mediator persisting into the 

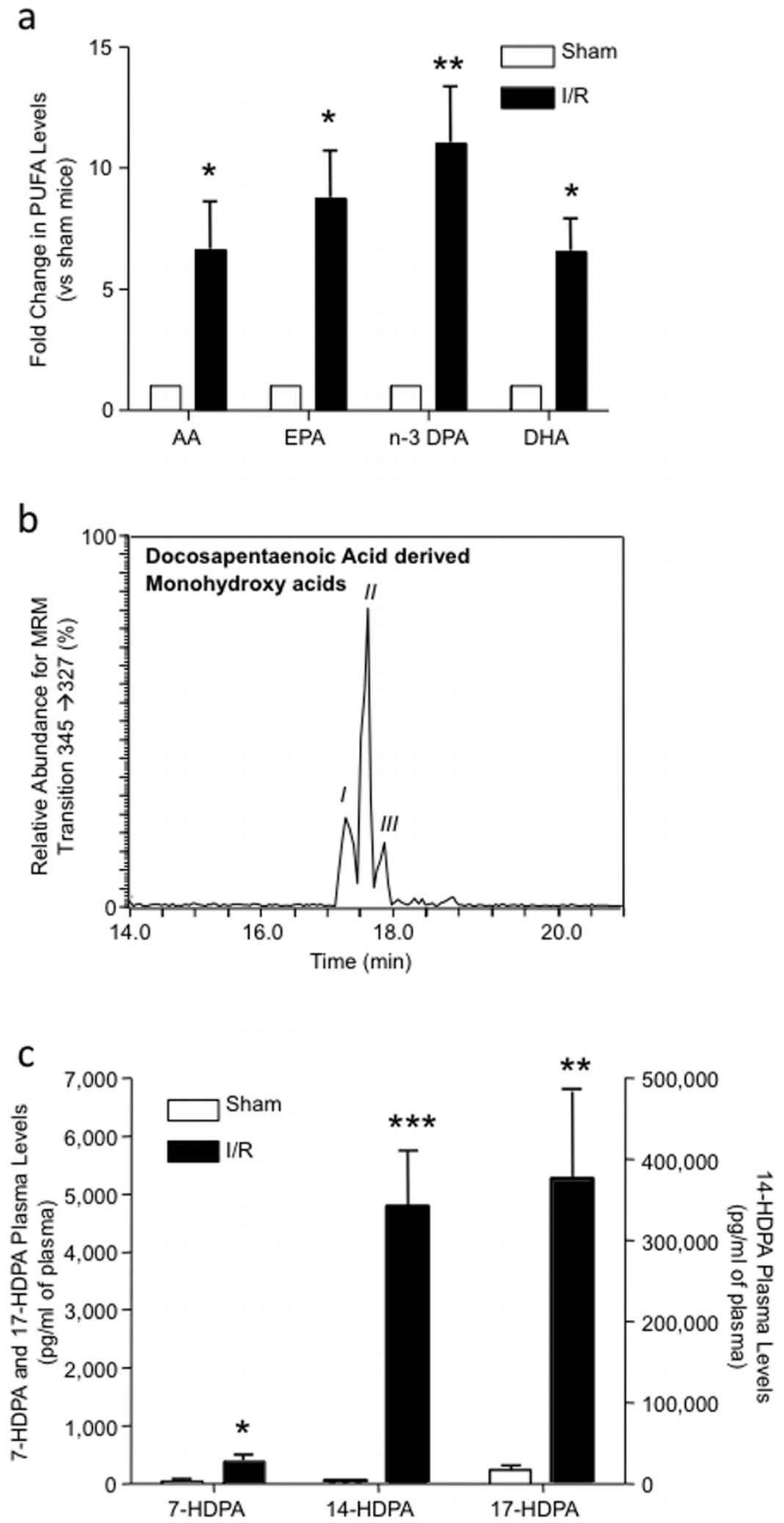

Figure 2 n-3 DPA levels increase in acute inflammation and is converted to novel products in vivo. Mice were subjected to ischemia reperfusion injury (see Methods for details) at $2 \mathrm{~h}$ of reperfusion, blood was collected via cardiac puncture and plasma was obtained by centrifugation, products were extracted and monohydroxy n-3 DPA levels were assessed by lipid mediator metabololipidomics. (a) Plasma polyunsaturated fatty acid levels;

(b) Representative chromatographs obtained by Multiple Reaction Monitoring $(\mathrm{MRM})$ of the parent ion $\left(\mathrm{Q}_{1}\right) \mathrm{m} / \mathrm{z} 345$ and a diagnostic daughter ion $\left(\mathrm{Q}_{3}\right)$ $\mathrm{m} / \mathrm{z}$ 327. (c) Monohydroxy-containing levels in plasma of sham mice and mice subjected to I/R. Results for a and $\mathrm{c}$ are mean $\pm S E M$. $\mathrm{n}=4$. Results for $\mathrm{b}$ are representative of $\mathrm{n}=4 .{ }^{*} P<0.05 ; * * P<0.01 ; * * P<0.01$ vs. sham mice. 

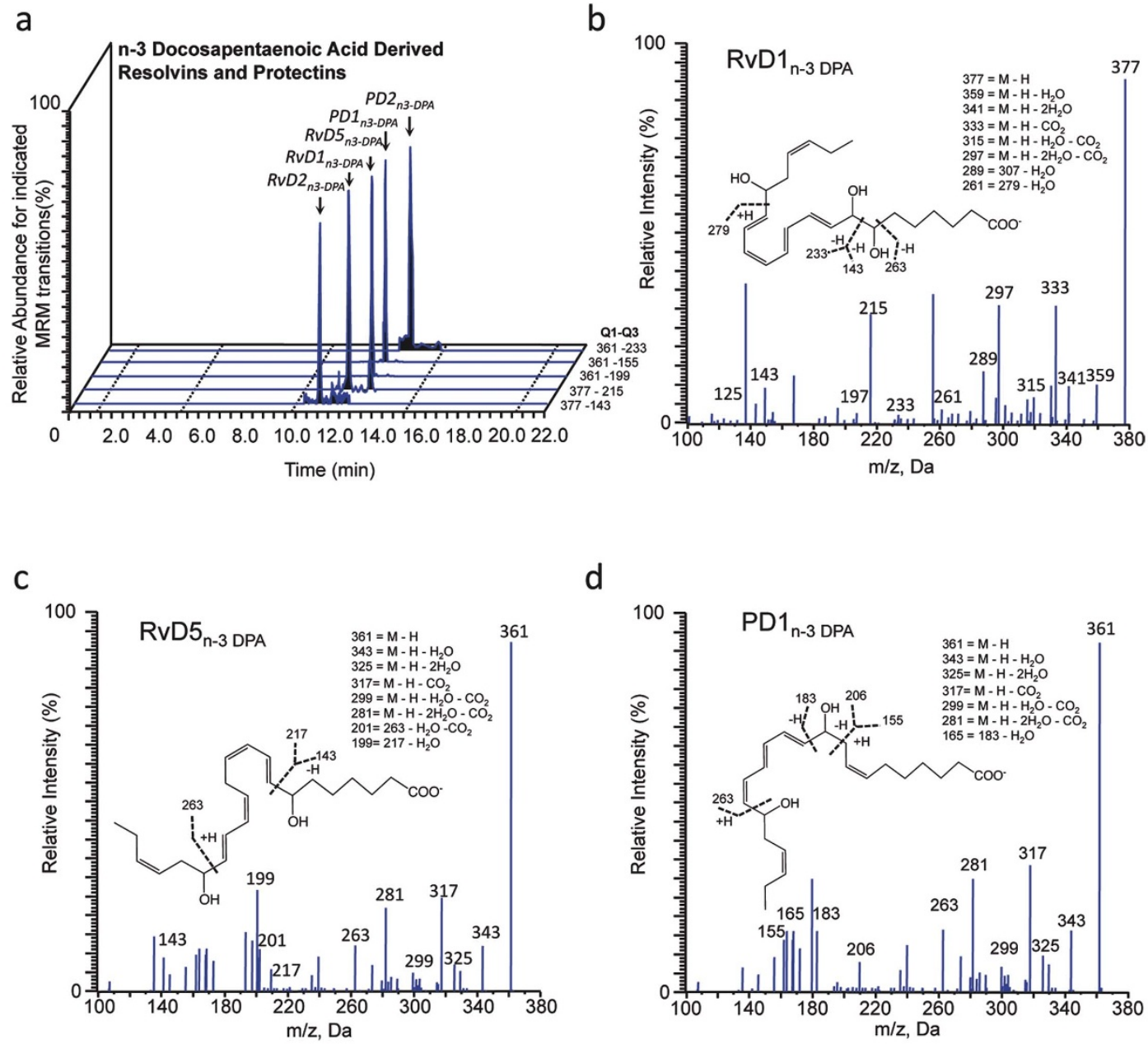

Figure 3 Identification of novel endogenous n-3 DPA pro-resolving mediators. Mice were subjected to ischemia reperfusion injury (see Methods and Fig. 2 for details). Two h into reperfusion, blood was collected and lipid mediators identified by lipid mediator metabololipidomics. (a) Representative chromatographs obtained by Multiple Reaction Monitoring of the parent ion $\left(\mathrm{Q}_{1}\right)$ and a diagnostic daughter ion $\left(\mathrm{Q}_{3}\right)$ in the MS-MS of n3-DPA resolvins, protectins and maresins. Representative MS-MS spectra used for identification of (b) RvD1 $1_{n-3}$ DPA $(c)$ RvD5 $5_{n-3}$ DPA and (d) PD1 $1_{n-3}$ DPA. Results are representative of $\mathrm{n}=4$.

resolution phase. In this experimental setting, which characterizes the initiation and resolution phases of the inflammatory response $\mathrm{e}^{25}$, we determined the profile of novel n-3 DPA products in order to temporally stage each of these new products within the self-limited inflammatory response. Here we found that endogenous production of $\mathrm{RvD}_{\mathrm{n}-3}$ DPA and $\mathrm{PD} 2_{\mathrm{n}-3}$ DPA displayed a bi-phasic profile, reaching a maximum during peak neutrophil infiltration and late into resolution (Figure $4 c, d$ ). $P D 1_{n-3}$ DPA,$M a R 2_{n-3}$ DPA and $M a R 3_{n-3}$ DPA levels were each found to reach a maximum at the $4 \mathrm{~h}$ interval and gradually decreased over the next $20 \mathrm{~h}$ (Figure $4 \mathrm{~d}, \mathrm{e})$. The peak in exudate RvD2 $2_{n-3}$ DPA levels coincided with the onset of resolution (the point where $\mathrm{PMN}$ levels reach $\sim 50$ of $\mathrm{T}_{\text {max }}$ ). $\mathrm{RvD} 5_{\mathrm{n}-3 \mathrm{DPA}}$ levels were found to gradually increase over the course of inflammationresolution, with a maximum being reached late in the resolution phase. The n-3 DPA product corresponding to $M a R 1_{n-3}$ DPA gave levels that were elevated in the peritoneum of naive mice, where upon challenge with zymosan these levels drastically decreased. Also, MaR $1_{n-3}$ DPA accumulated late during resolution (Figure 4e). These results establish the endogenous production of novel n-3 DPA resolvins, protectins and maresins during acute inflammation and stage their formation primarily within the self-limited inflammatory response.

Specialized pro-resolving mediators from n-3 DPA exert potent anti-inflammatory actions in vivo. We next tested whether these novel structures carried bioactivity. Intravenous administration of $100 \mathrm{ng}$ of $\mathrm{RvD} 1_{\mathrm{n}-3}$ DPA and $\mathrm{RvD} 2_{\mathrm{n}-3}$ DPA significantly reduced neutrophil recruitment into the peritoneum (A; $\sim 45 \%$; Fig. $5 \mathrm{a}$ ). In these experiments, we also found that the novel tri-hydroxycontaining n-3 DPA products significantly reduced exudate levels of the pro-inflammatory cytokines Interleukin (IL) $6 \quad(\sim 55 \%$; Fig. 5b) and monocyte chemoattractant protein (MCP)-1 ( $55 \%$; Fig. 5c). Administration of the di-hydroxy-containing n-3 DPA products from both 17-hydroperoxy-DPA (HpDHA; RvD5n-3 DPA and $\mathrm{PD} 1_{\mathrm{n}-3}$ DPA $\left.; \mathrm{B} ; \sim 47 \%\right)$ and $14-\mathrm{HpDPA}\left(\mathrm{MaR}_{\mathrm{n}-3}\right.$ DPA and $\mathrm{MaR}_{\mathrm{n}-3}$ DPA; C; $\sim 50 \%$ ) also significantly reduced PMN recruitment and pro-inflammatory cytokine levels in these exudates (Fig. 5a-c).

Human leukocytes produce n-3 DPA immunoresolvents. Having found that these products are produced in murine systems, we next sought evidence for their production by human leukocytes. Assessment of methyl formate fractions obtained from activated human peripheral blood neutrophils incubated with n-3 DPA by lipid mediator metabololipidomics profiling gave products that displayed chromatographic and MS-MS spectra consistent with resolvins, protectins and maresins that carry the n-3 DPA backbone (Fig. 5a). Assessment of the UV absorbance spectra and MS-MS fragments for each of the products gave fragment ions that 
a

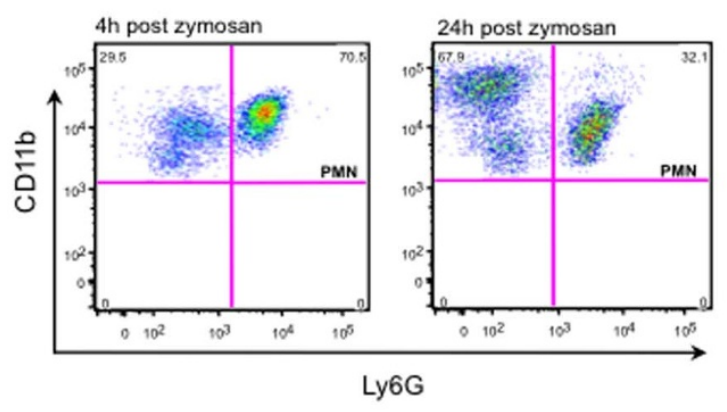

b

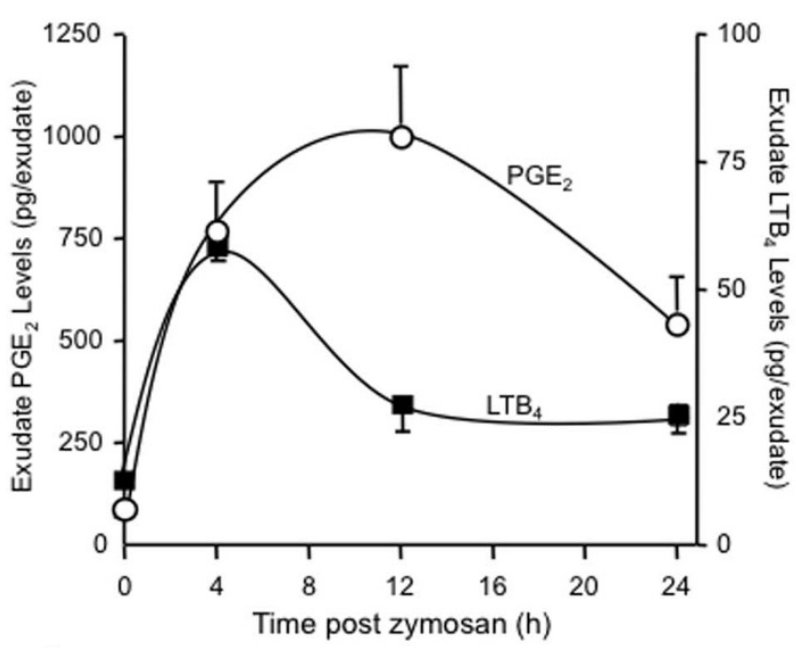

d

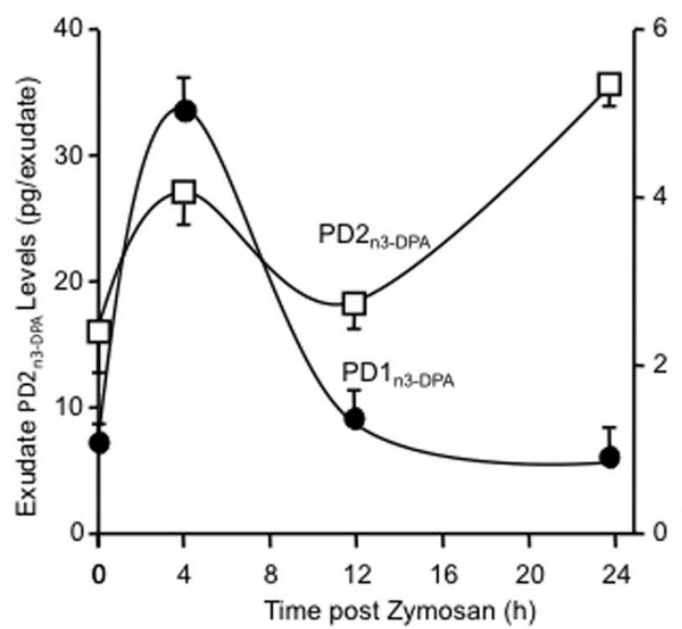

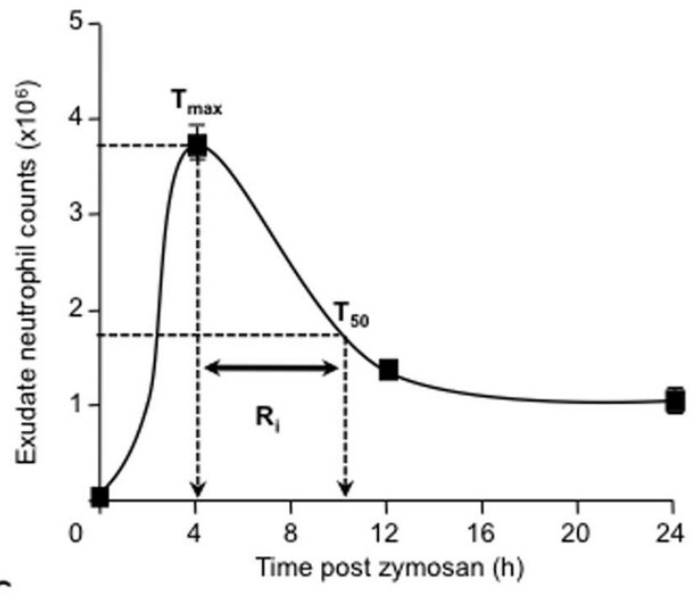

C

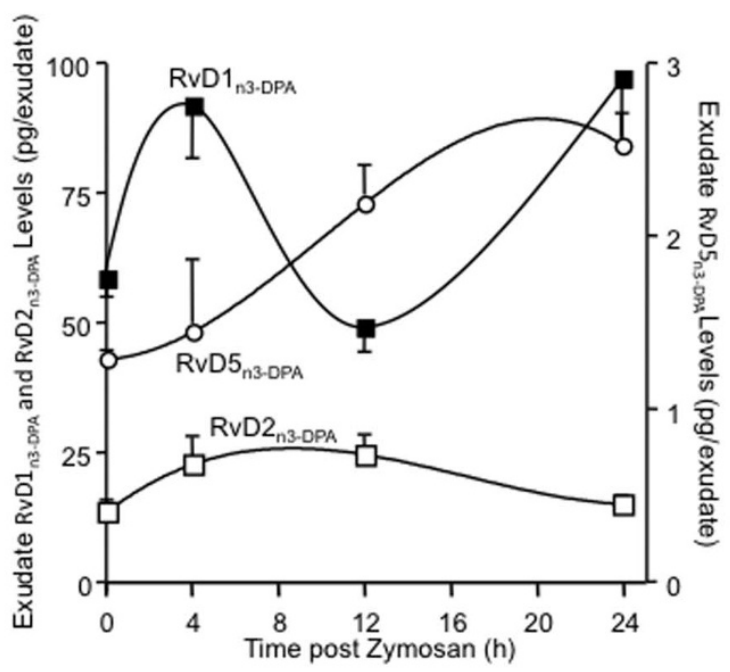

e

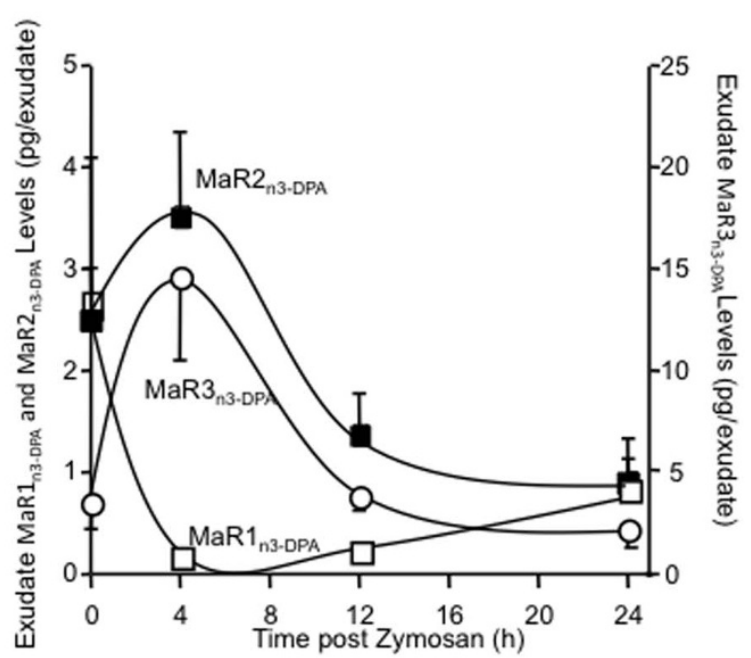

Figure 4 | Self-limited inflammation: Endogenous formation of novel immunoresolvents from n-3 DPA. Mice were treated with 0.1 mg zymosan i.p.; after the indicated time intervals peritoneal exudates were collected. (a) Exudate leukocyte counts obtained by light microscopy and flow cytometry. Exudate levels for (b) prostaglandin (PG) $\mathrm{E}_{2}$ and leukotriene (LT) $\mathrm{B}_{4}$; (c) resolvins, (d) protectins and (e) maresins were measured by lipid mediator metabololipidomics. Results are mean \pm SEM. $\mathrm{n}=4$ mice per time point.

were consistent with $\mathrm{RvD}_{\mathrm{n}-3}$ DPA with $\mathrm{m} / \mathrm{z} 377$ [M-H], m/z 359 $\left[\mathrm{M}-\mathrm{H}-\mathrm{H}_{2} \mathrm{O}\right]$ and $\mathrm{m} / \mathrm{z} 333\left[\mathrm{M}-\mathrm{H}-\mathrm{CO}_{2}\right]$ (Fig. 5a and Supplementary Fig. 3a, b). Additional diagnostic ions were identified at $\mathrm{m} / \mathrm{z} 247, \mathrm{~m} / \mathrm{z}$ 143 and $\mathrm{m} / \mathrm{z} 279$ consistent with the presence of hydroxy groups at carbon positions 7,16 and 17 . In the tri-hydroxy chromatographic regions, we also identified $\mathrm{RvD}_{\mathrm{n}-3}$ DPA as demonstrated by its characteristic fragmentation pattern and UV absorbance spectra, consistent with hydroxyl groups at the carbon 7,8 and 17 positions (Fig. 3a, b and Supplementary Fig 3c, d). Assessment of the UV absorbance and MS-MS spectra in the di-hydroxy region 

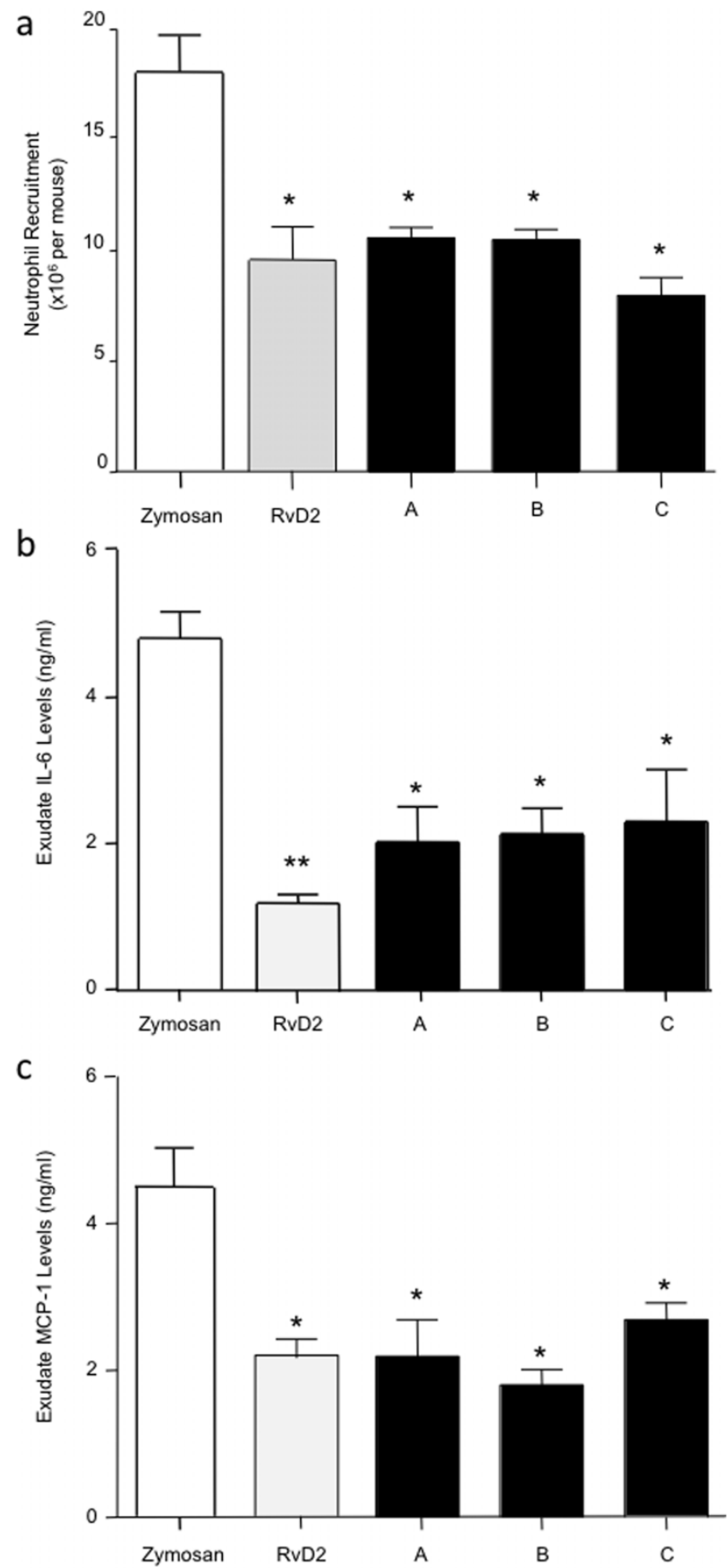

Figure $5 \mid$ n-3 DPA immunoresolvents display potent anti-inflammatory actions in vivo. The indicated n-3 DPA-derived product mixtures were administered by intravenous injection $10 \mathrm{~min}$ prior to the intraperitoneal administration of zymosan $(0.1 \mathrm{mg}, 500 \mu \mathrm{lPBS})$ to 6-8-week-old male FvB mice. At $4 \mathrm{~h}$, peritoneal exudates were collected and the (a) number of infiltrated neutrophils was assessed by light microscopy and flow cytometry. Exudate levels for the pro-inflammatory mediators (b) IL6 and (c) MCP- 1 were determined by cytokine array. The ratio of RvD1 $1_{n-3 D P A}$ to $\operatorname{RvD} 2_{n-3}$ DPA was $\sim 3: 1(\mathrm{~A})$, the ratio of MaR $1_{n-3}$ DPA to MaR2 $2_{n-3}$ DPA was $\sim 4: 1$ (B); the ratio of RvD5 $5_{n-3}$ DPA to PD1 $1_{n-3}$ DPA was $\sim 9: 1$ (C). Results are mean \pm SEM. $\mathrm{n}=4$. $^{*} P<0.05,{ }^{* *} P<0.01$ vs. zymosan mice.

revealed the presence of RvD5 $n-3$ DPA (Fig. 3a, c and Supplementary Fig 3e, f), PD1 $1_{n-3}$ DPA (Fig. 3a and Supplementary Fig. 5a, b) and $\mathrm{MaR}_{\mathrm{n}-3}$ DPA (Fig. 3a and Supplementary Fig. 5e, f). Using LM metabololipidomics we also found that human monocyte-derived macrophages produce resolvins, protectins and maresins from endogenous n-3 DPA $(n=3)$. These findings demonstrate that human leukocytes can convert both exogenous as well as endogenous n-3 DPA to novel n-3 DPA immunoresolvents.

n-3 DPA products exert anti-inflammatory and pro-resolving actions on human leukocytes. We next investigated whether these novel n-3 DPA products retained their leukocyte directed antiinflammatory and pro-resolving actions when incubated with human leukocytes. A key step in neutrophil recruitment to the site of inflammation is firm adhesion to the vascular endothelium ${ }^{26}$. Incubation of neutrophils with $\mathrm{RvD}_{\mathrm{n}-3} \mathrm{DPA}$ and $\mathrm{RvD} 2_{\mathrm{n}-3} \mathrm{DPA}$, (A;

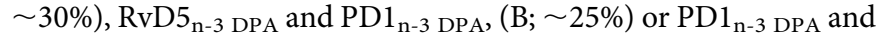
$\mathrm{PD} 2 \mathrm{n}-3$ DPA $(\mathrm{C} ; \sim 22 \%)$ led to a significant reduction in neutrophil adhesion to TNF- $\alpha$ activated endothelial cells to a similar extent as RvD2 ( $\sim 30 \%$; Fig. $7 \mathrm{a})$, a potent pro-resolving mediator ${ }^{27}$.

Neutrophil-endothelial cell adhesion is mediated by adhesion molecules expressed on both the endothelial and neutrophil surface that are up-regulated during inflammation ${ }^{26}$. One of these adhesion molecules is Intercellular Adhesion Molecule 1 (ICAM-1/CD54) expressed on endothelial cells ${ }^{28}$. Incubation of each of the n-3 DPA products with endothelial cells prior to incubation with TNF- $\alpha$ also led to a significant reduction in endothelial cell ICAM-1 expression ( 25-40\%; Fig. 5b).

We next questioned whether the n-3 DPA pro-resolving mediators regulated human peripheral blood leukocyte recruitment to a chemotactic stimulus. Incubation of human leukocytes with RvD2 led to a significant reduction in neutrophil $(\sim 42 \%)$ recruitment towards an IL-8 gradient. When human neutrophils were incubated with RvD1 $1_{n-3}$ DPA and RvD2 $2_{n-3}$ DPA $(1 \mathrm{nM})$, we also found a significant reduction in neutrophil chemotaxis $(\sim 45 \%)$ towards an IL-8 gradient (Fig. 7b). Incubation of human neutrophils with $1 \mathrm{nM}$ of the di-hydroxy containing products (i.e. $\mathrm{RvD}_{\mathrm{n}-3 \mathrm{DPA}}$ and $\mathrm{PD} 1_{\mathrm{n}-3 \mathrm{DPA}}, \mathrm{C}$; or MaR1 $1_{\mathrm{n}-3}$ DPA and MaR2 $2_{\mathrm{n}-3}$ DPA, $\left.\mathrm{D}\right)$ also led to a significant reduction in neutrophil chemotaxis $(\sim 40-75 \%)$ towards IL8 (Fig. $7 \mathrm{~b}$ ).

Because macrophage clearance of cellular debris and apoptotic cells is a critical process in promoting the resolution of acute inflammation $^{1}$, we next incubated human monocyte-derived macrophages with RvD2, which led to a significant increase in the uptake of opsonized zymosan particles ( $~ 50 \%$; Fig. $7 \mathrm{c})$. Addition of RvD5 $n-3$ DPA and $\mathrm{PD} 1_{\mathrm{n}-3 \mathrm{DPA}},(1 \mathrm{nM})$ to macrophages each also led to a significant increase $(\sim 70 \%)$ in macrophage phagocytosis of fluorescently labeled zymosan (Fig. 7c). Macrophage incubations with MaR $1_{n-3}$ DPA and $\mathrm{MaR} 2_{\mathrm{n}-3}$ DPA $(\sim 55 \%)$ or $\mathrm{RvD} 1_{\mathrm{n}-3}$ DPA and $\mathrm{RvD} 2_{\mathrm{n}-3}$ DPA $(\sim 45 \%)$ also gave significant increases in macrophage uptake of fluorescently labeled zymosan (Fig. 7c). These results demonstrate that the novel n-3 DPA mediators exert potent anti-inflammatory and pro-resolving actions with human leukocytes limiting human neutrophil recruitment and macrophage phagocytosis, key processes in promoting resolution and by definition those assigned to proresolving mediators ${ }^{1,2}$.

\section{Discussion}

In the present report, using lipid mediator metabololipidomics, we identified novel n-3 DPA-derived products and staged their biosynthesis during self-limited inflammation in inflammatory exudates. With murine models of acute inflammation and human leukocytes, we determined their anti-inflammatory, pro-resolving and tissue protective actions, defining these novel n-3 DPA products as immunoresolvents.

In humans, circulating n-3 DPA levels do not appear to be directly associated with dietary intake, unlike other n-3 EFA including DHA and $\mathrm{EPA}^{21,29}$, thus suggesting in humans a primary endogenous metabolic origin for n-3 DPA. Along these lines, a recent genome wide association study with more then 8500 participants from various ethnicities demonstrated that elevated plasma n-3 DPA levels are 
associated with single nucleotide polymorphisms in the genes encoding for the fatty acid elongase 2 (ELOVL2) and glucokinase regulatory protein $(G C K R)^{21}$. This increase in n-3 DPA levels is also associated with a reduction in circulating DHA levels. Of interest, circulating n-3 DPA has been associated with protection from cardiovascular disease $\mathrm{e}^{30-32}$. Hence, our present findings indicating that $n-3$ DPA is a precursor to new potent bioactive products may have wide implications in individuals carrying elevated circulating levels of n-3 DPA.

Ischemia-reperfusion injury is of considerable consequence in the pathology of many diseases including periodontal disease, arthritis and stroke as well as being of relevance during surgical procedures, in particular those involving extremities, causing aberrant leukocyte activation that results in local and remote tissue and organ damage ${ }^{33}$. Neutrophils in these settings play pivotal roles in the perpetuation of reperfusion injury giving rise to second organ injury ${ }^{33}$. However, phagocytes, including neutrophils when appropriately activated, are also instrumental in orchestrating resolution processes via their temporal production of pro-resolving mediators in resolving exudates ${ }^{14,15,34,35}$. Hence our findings indicating that n-3 DPA products obtained from phagocytes collected from self-resolving exudates display potent protective actions from second organ injury during ischemia reperfusion have implications in a wide range of human pathologies. Indeed these products markedly reduced both local tissue damage and neutrophil infiltration into the lungs (Fig. 1), a hallmark of second organ injury, to a similar extent as the DHA-derived resolvin D1, a known potent pro-resolving mediator ${ }^{9}$.

The formation of platelet-leukocyte aggregates in the vasculature is also a component of many inflammatory disorders including stroke, sepsis and hypertension. Formation of these microcellular aggregates enhances the production of a number of pro-inflammatory cytokines including IL- 8 and MCP-1 as well as increases the levels of platelet aggregating factor, a potent pro-inflammatory lipid mediator (for a review, see ref. 24). In this context, elevated levels of circulating platelet-leukocyte aggregates are proposed as an early marker for acute myocardial infarction and are increasingly regarded as a cardiovascular risk factor ${ }^{36}$. In the present studies, administration of phagocyte-derived n-3 DPA products led to a reduction in circulating platelet-neutrophil aggregates following ischemia

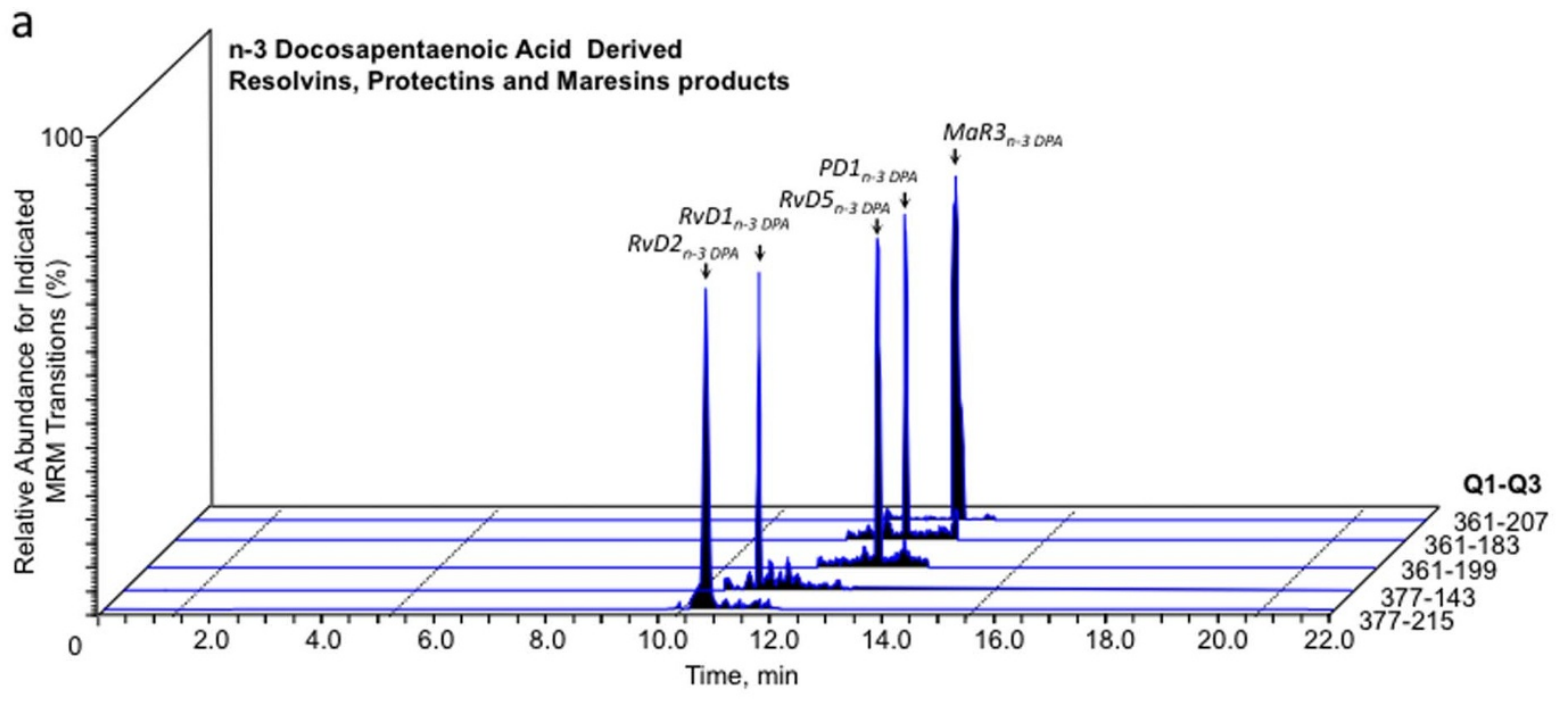

b

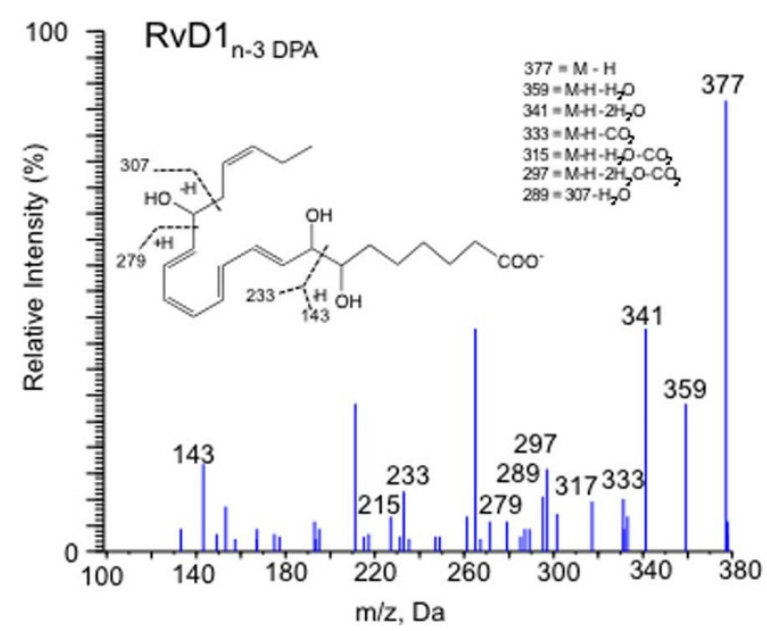

C

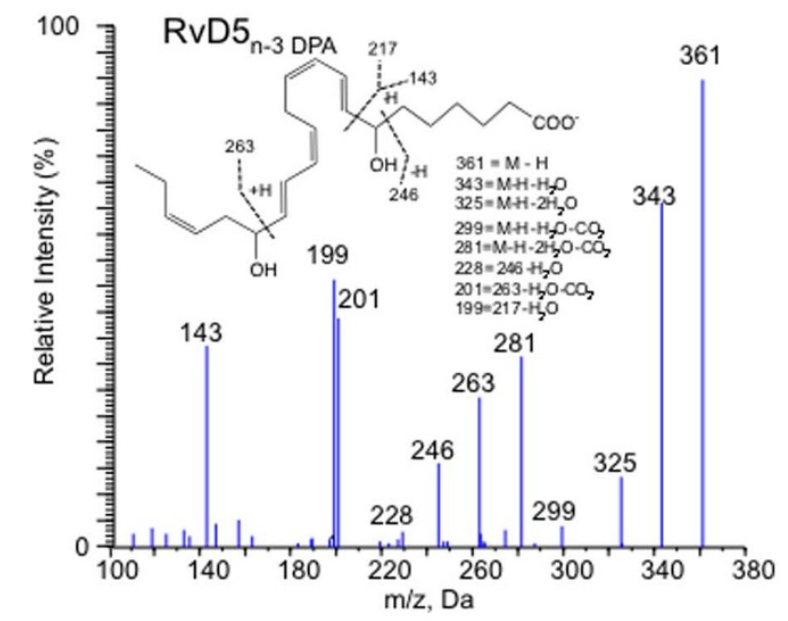

Figure 6 Human neutrophils produce novel n-3 DPA-derived immunoresolvents. Human neutrophils were prepared from peripheral blood (see Methods for details), suspended in DPBS $\left(80 \times 10^{6} / \mathrm{ml}\right)$ and incubated with serum treated zymosan $(0.1 \mathrm{mg})$ and $\mathrm{n}-3 \mathrm{DPA}\left(1 \mu \mathrm{M}, 30 \mathrm{~min}, 37^{\circ} \mathrm{C}\right.$, $\mathrm{pH}$ 7.45); incubations were stopped with ice-cold methanol and products assessed by lipid mediator metabololipidomics. (a) Representative chromatographs obtained by Multiple Reaction Monitoring of the parent ion $\left(\mathrm{Q}_{1}\right)$ and a diagnostic daughter ion $\left(\mathrm{Q}_{3}\right)$ in the MS/MS. Representative MS/MS spectra used for identification of (b) $R v D 1_{n-3}$ DPA and (c) $R v D 5_{n-3}$ DPA. Results are representative of $n=4$. 
a

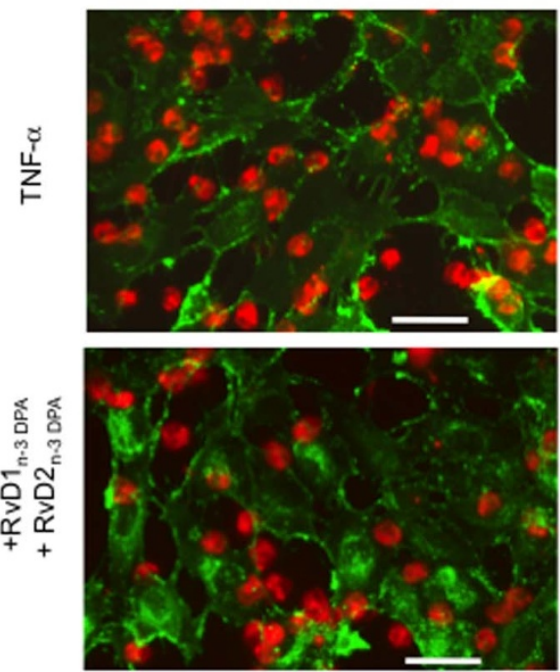

b

Reduction in Human Neutrophil Chemotaxis (\% of Veh Control)

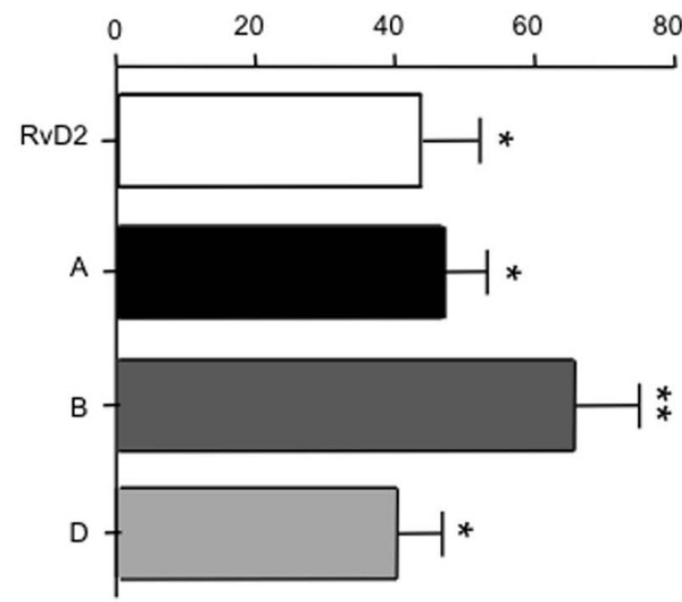

Reduction in Human Neutrophil Adhesion (\% of Veh Control)

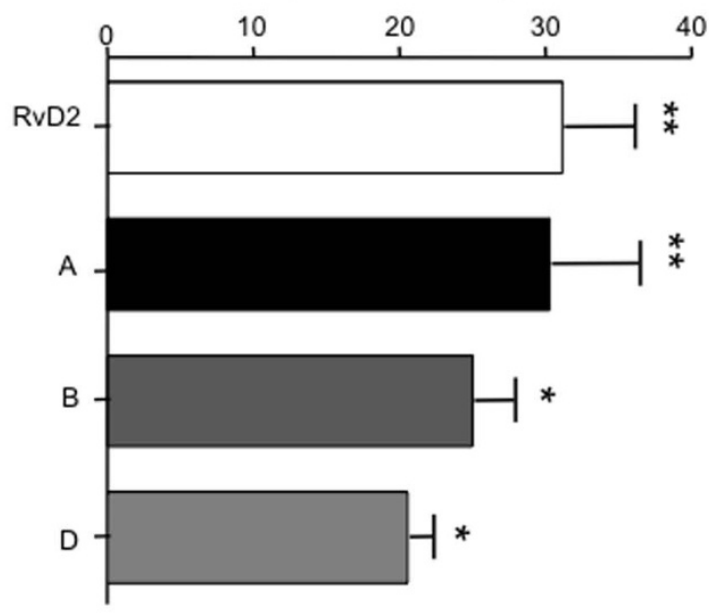

C

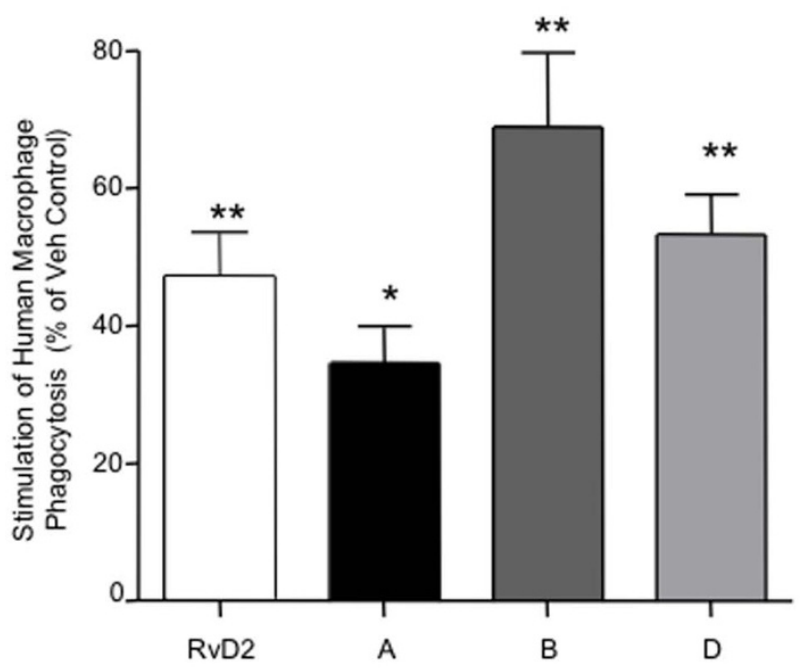

Figure $7 \mid$ Reduction in human neutrophil chemotaxis, neutrophil-endothelia cell adhesion and stimulation of macrophage phagocytosis by $\mathbf{n}-3$ DPA-derived immunoresolvents. (a) Left panel: micrographs depict PKH26-labeled neutrophils adherent to WGA-Alexafluor ${ }^{\circledR}$ 488-labeled HUVEC stimulated with TNF- $\alpha\left(10 \mathrm{ng} / \mathrm{ml}, 4 \mathrm{~h}, 37^{\circ} \mathrm{C}, 0.1 \%\right.$ FCS $)$ with or without $\mathrm{n}-3$ DPA resolvins $\left(1 \mathrm{nM}, 15 \mathrm{~min}, 37^{\circ} \mathrm{C}, \mathrm{pH} 7.45 ; \times 40 \mathrm{magnification}\right)$. Right panel: Fluorescently labeled human neutrophils were incubated with vehicle $(0.1 \% \mathrm{EtOH}$ in PBS) or n-3 DPA products. These were then added to TNF- $\alpha$ stimulated HUVEC and incubated for $30 \mathrm{~min}\left(37^{\circ} \mathrm{C}\right)$, non-adherent cells were washed and extent of neutrophil adhesion assessed using a SpectraMax M3 Plate reader. (b) Neutrophils were incubated with vehicle ( $0.1 \%$ EtOH in PBS) or n-3 DPA products ( $\left.1 \mathrm{nM}, 15 \mathrm{~min}, 37^{\circ} \mathrm{C}, \mathrm{pH} 7.45\right)$ prior to loading on ChemoTx chambers and assessing chemotaxis towards IL-8 $\left(100 \mathrm{ng} / \mathrm{ml}, 90 \mathrm{~min}, 37^{\circ} \mathrm{C}, \mathrm{pH} 7.45\right)$. (c) Macrophages were incubated with vehicle $(0.1 \%$ EtOH in PBS) or $\mathrm{n}-3$ DPA products $\left(1 \mathrm{nM}, 15 \mathrm{~min}, 37^{\circ} \mathrm{C}, \mathrm{pH} 7.45\right)$ prior to addition of fluorescently labeled zymosan ( $1: 10$ macrophages to zymosan). After $60 \mathrm{~min}\left(37^{\circ} \mathrm{C}, \mathrm{pH} 7.45\right)$, the incubation was stopped, extracellular fluorescence quenched using trypan blue and phagocytosis assessed using a SpectraMax M3 Plate reader. The ratio of $\mathrm{RvD}_{\mathrm{n}-3 \mathrm{DPA}}$ to $\mathrm{RvD} 2_{\mathrm{n}-3 \mathrm{DPA}}$ (A) was $\sim 3: 1$; the ratio of $\mathrm{RvD} 5_{\mathrm{n}-3} \mathrm{DPA}$ to PD1 $1_{\mathrm{n}-3} \mathrm{DPA}$ (B) was $\sim 9: 1$; the ratio of $\mathrm{PD} 1_{\mathrm{n}-3 \mathrm{DPA}}$ to $\mathrm{PD} 2_{\mathrm{n}-3 \mathrm{DPA}}(\mathrm{C})$ was $\sim 1: 5$; the ratio of MaR $1_{\mathrm{n}-3 \mathrm{DPA}}$ to MaR $2_{\mathrm{n}-3 \mathrm{DPA}}(\mathrm{D})$ was $\sim 4: 1$. Results are mean $\pm \mathrm{SEM}$. $\mathrm{n}=4$ independent neutrophil and macrophage preparations $\left({ }^{*} P<0.05 ;{ }^{*} P<0.05\right.$ vs. vehicle incubated cells). Bar $=50 \mu \mathrm{M}$.

reperfusion injury. In addition, we also found a substantial reduction in plasma pro-inflammatory eicosanoid levels including $\mathrm{LTB}_{4}$ and $\mathrm{TxB}_{2}$ (Fig. 1), actions that were comparable to those afforded by DHA-derived RvD1. Therefore, these results demonstrate that $n-3$ DPA products display potent systemic anti-inflammatory and tissue protective actions.

Phagocytes carry lipoxygenase enzymes that are involved in the biosynthesis of pro-resolving mediators ${ }^{14,15,35}$. These enzymes convert their substrate in a stereospecific manner, inserting molecular oxygen predominantly in the $S$ configuration ${ }^{5,34}$. In the present study we found that the systemic levels of n-3 DPA and the new n-3 DPA products (i.e. 17-HDPA, 14-HDPA and 7-HDPA) were each elevated during acute inflammation in vivo (Fig. 2). In addition, chiral lipidomics of these n-3 DPA products demonstrated that the hydroxy groups in these products were predominantly in the $S$ configuration suggesting that each was produced via lipoxygenases (Supplementary Fig. 2). Targeted metabololipidomics demonstrated that n-3 DPA is further oxygenated, in both murine in vivo and isolated human leukocytes, to products that are congenerous of the DHA-derived pro-resolving mediators (Figs. 3, 6). In addition, endogenous levels of these novel n-3 DPA mediators were temporally regulated during self-limited inflammation suggesting that each may possess distinct roles in the regulation of inflammationresolution and in organ protection (Fig. 4). 

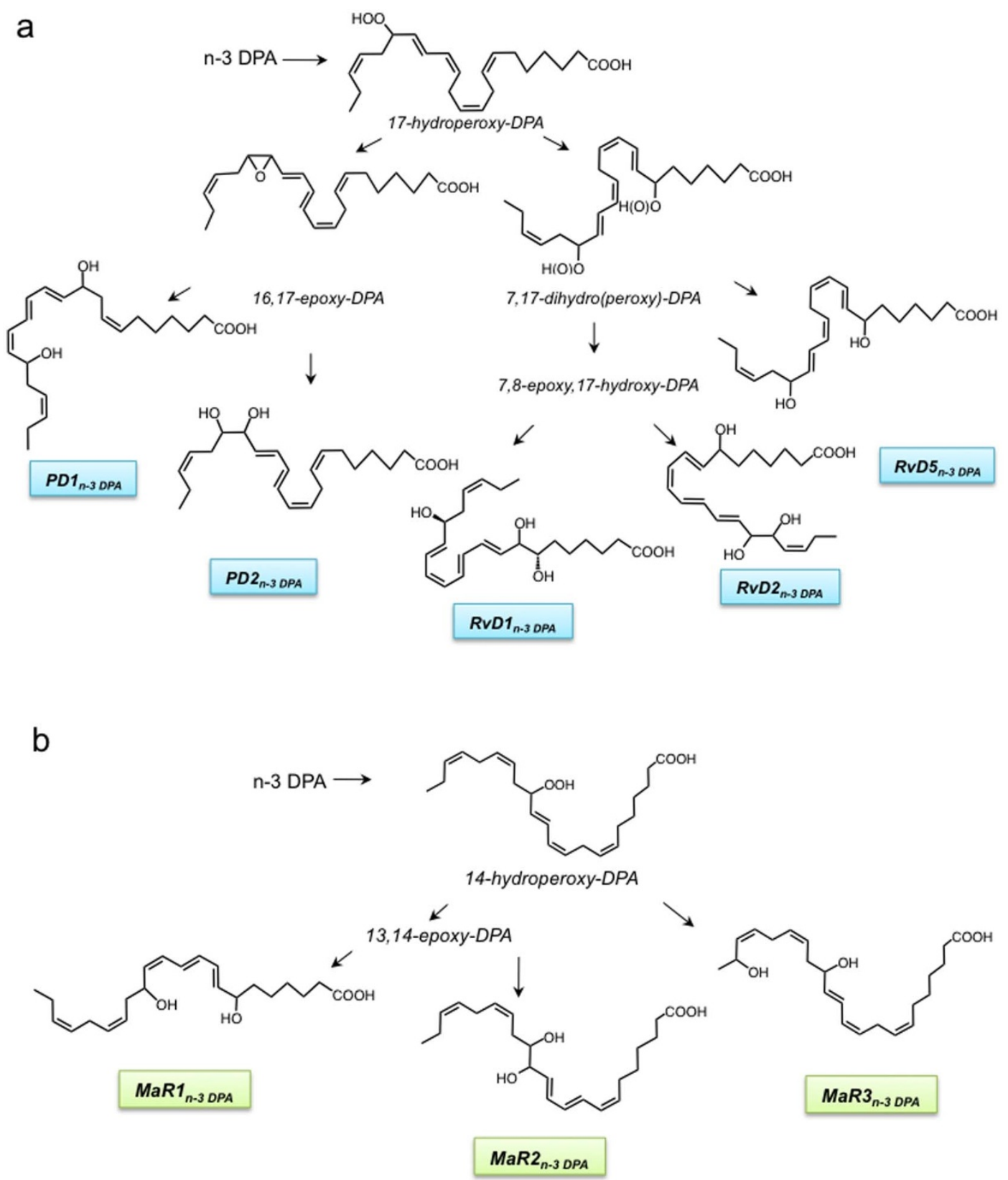

Anti-inflammatory, Pro-resolving, Organ Protective

Figure 8 Biosynthetic schemes proposed for novel n-3 docosapentaenoic acid products and their actions. At the site of injury, n-3 DPA is converted to (a) 17-HpDPA that undergoes further conversion by lipoxygenation to the $\mathrm{n}-3$ DPA resolvins. 17-HpDPA is also a substrate for enzymatic conversion to an epoxide intermediate that is next enzymatically hydrolyzed to the n-3 DPA protectin structures. (b) n-3DPA is also converted to 14-lipoxygenation to yield 14-HpDPA that is further converted to an epoxide intermediate and then enzymatically hydrolyzed to MaR1 $1_{\mathrm{n}-3} \mathrm{DPA}$ and/or MaR2 $2_{\mathrm{n}-3} \mathrm{DPA}$. 14 -HpDPA can also undergo a second oxygenation at the omega -1 position to yield the MaR3 $3_{n-3}$ DPA. Note that each product is depicted in the $17 S$ and $14 S$ configuration based on the results obtained from chiral lipidomics that indicated $\mathrm{S}$ as the predominate form of each but may also carry $17 \mathrm{R}$ as well as $14 \mathrm{R}$ chirality from lipoxygenase reactions as these lesser components (see Supplementary Fig. 2 and text for details). The complete stereochemistries of these novel mediators remain to be established and are depicted in their likely configuration based on biogenic synthesis (see Supplementary Figures $3-5$ for retention times, UV and MS-MS for each of these mediators).

In light of the results from the present studies in conjunction with earlier mechanisms proposed for the biosynthesis of the Dseries resolvins, protectins $s^{15,34}$ and maresins ${ }^{35}$, the pathways for the novel n-3 DPA immunoresolvent are illustrated in Fig. 8. In this proposed scheme, n-3 DPA is first converted via 17 lipoxygenation to 17-hydroperoxy-8Z,10Z,13Z,15E,19Z-docosapentaenoic acid (17-HpDPA). This intermediate can next undergo a second lipoxygenation by 5-lipoxygenase-like reaction to yield the 7,8 , 17-trihydroxy-9,11,13,15E,19Z-docosapentaenoic acid (RvD1 $\left.1_{n-3 D P A}\right)$, 7, 16, 17-trihydroxy-8,10,12,14E,19Z-docosapentaenoic acid (RvD2 $\left.{ }_{\mathrm{n}-3 \mathrm{DPA}}\right)$ and/or 7,17-trihydroxy-8E,10,13,15E,19Z-docosapentaenoic acid (RvD5 $\left.{ }_{n-3 D P A}\right)$. In addition, 17-HpDPA can undergo 
enzymatic conversion to an epoxide intermediate that is next enzymatically hydrolyzed to either 10,17-dihydroxy-7Z,11,13,15,19Zdocosapentaenoic acid (PD1 $\left.1_{\mathrm{n}-3 \mathrm{DPA}}\right)$ or 16,17-dihydroxy-7Z,10,13, 14,19Z- docosapentaenoic acid (PD2 $\left.2_{\mathrm{n}-3 \mathrm{DPA}}\right)$. In a parallel pathway, the arachidonate 12-lipoxygenase coverts $n-3$ DPA to 14-hydroperoxy-7Z,10 Z,12E,16Z,19Z-docosapentaenoic acid (14-HpDPA) that is further converted to an epoxide intermediate and then enzymatically hydrolyzed to yield 7,14-dihydroxy-8,10,12,16Z,19Zdocosapentaenoic acid (MaR1 $1_{\mathrm{n}-3}$ DPA $)$ or 13,14-dihydroxy-7Z,9,11, $16 Z, 19 Z$-docosapentaenoic acid $\left(\mathrm{MaR}_{\mathrm{n}-3} \mathrm{DPA}\right)$. Alternatively, this $14-H p D P A$ can undergo a second oxygenation at the omega -1 position to yield 14, 21-dihydroxy-7Z,10Z,12E,16Z,19Z-docosapentaenoic acid (MaR3 $\left.{ }_{\mathrm{n}-3 \mathrm{DPA}}\right)$. Of note, since we identified the $S$ isomer of both 17-HDPA and 14-HDPA, the reduction products of 17HpDPA and 14-HpDPA, as the major products in inflamed tissues (Supplementary Fig. 2), it is highly likely that the stereochemistry at these positions is retained in the biosynthesis of the novel n-3 DPAderived resolvins, protectins and maresins. Of note, it is conceivable that the $R$-containing diastereomers of the n-3 DPA resolvins, protectins and maresins may also be of biological relevance in inflammation-resolution, since they were also obtained via lipoxygenation reaction albeit to lesser proportions than their corresponding $\mathrm{R}-\mathrm{con}$ taining products (see Supplementary Fig. 2).

Excessive neutrophil activation and infiltration to the inflamed site can be detrimental since it may lead to further tissue damage and propagation of the inflammatory response $e^{4,13}$. When administered in vivo, RvD1 $1_{n-3}$ DPA,$R v D 2_{n-3}$ DPA,$R v D 5_{n-3}$ DPA,$P D 1_{n-3}$ DPA,$M a R 1_{n-3}$ DPA, and $M a R 2_{n-3}$ DPA each reduced neutrophil infiltration in murine peritonitis (Fig. 5). These mediators also demonstrated potent cytokine counter-regulatory actions reducing exudate levels of IL-6 and MCP-1 (Fig. 5) to levels comparable with the pro-resolving mediator $\mathrm{RvD}^{27}$. The novel n-3 DPA immunoresolvents were also found to exert potent anti-inflammatory actions with human neutrophils and endothelial cells regulating central steps in the leukocyte recruitment cascades, reducing neutrophil chemotaxis and adhesion to endothelial cells (Fig. 7) as well as expression of the adhesion molecule ICAM1 by endothelial cells (Supplementary Fig. 6). Importantly, these mediators were found to enhance macrophage phagocytosis, a key

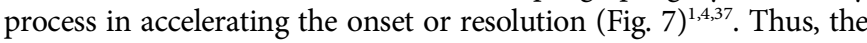
actions for each of these novel n-3 DPA products are in accord with the key characteristic that define an immunoresolvent, a property shared with their DHA resolution metabolome SPM counterparts.

Since mammals lack enzymes that can insert double bonds in either the n-3 or n-6 position to polyunsaturated fatty acids, the precursor molecules for the production of n-3 and n- 6 essential fatty acids, namely alpha linolenic acid (ALA) and linoleic acid (9Z,12Zoctadecadienoic acid), must be obtained via dietary intake ${ }^{12}$. In mammalian tissues, ALA is converted by elongation and desaturation to EPA and DHA. Linoleic acid on the other hand is converted, in a parallel pathway in humans, to arachidonic acid and subsequently to n-6 DPA ${ }^{10,12}$. Thus, while n-3 DPA and n-6 DPA each share the carbon 22 and five unsaturated double bonds, their structures are different because they are produced via separate biosynthetic pathways from chemically dissimilar precursors and each possesses distinct biophysical properties ${ }^{22}$. Earlier, oxygenated products obtained from n-6 DPA were found to reduce neutrophil recruitment in a model of dermal inflammation ${ }^{38}$, enhance macrophage phagocytosis $^{39}$, reduce ear swelling in a model of delayed type hypersensitivity $^{38}$ and protect against intestinal damage in a mouse model of intestinal inflammation ${ }^{39}$, albeit displaying lower potency then the $\mathrm{n}-3$ pro-resolving mediators ${ }^{1}$ as well as compared to the new n-3 DPA mediators described herein. This is in accord with the finding that even subtle differences in the stereochemistry of lipid mediators can result in dramatic changes in their potency ${ }^{34}$.

In summary, herein we established the structures of new n-3 DPAderived products, their formation from endogenous sources, staged the production of each of these novel mediators during-inflammation resolution and determined their anti-inflammatory, proresolving and tissue protective properties. These actions are characteristic of specialized pro-resolving mediators ${ }^{37}$ and define these novel n-3 DPA products as immunoresolvents. In view of the role of lipid mediators in inflammation and its timely resolution ${ }^{1,4,37}$, the n-3 DPA metabolome documented herein may mediate some of the beneficial actions associated with probiotic dietary supplementation $^{40}$. Moreover, these new n-3 immunoresolvents may also serve as a compensatory mechanism in people with elevated n-3 DPA levels and lower circulating DHA levels ${ }^{21}$ to compensate for losses in DHA SPM-regulated leukocyte-mediated tissue damage and timely resolution of acute inflammation.

\section{Methods}

Materials. Zymosan A, bovine serum albumin (BSA), Roswell Park Memorial Institute media 1640 (RPMI 1640), DPBS and Histopaque 1077-1 were purchased from Sigma-Aldrich. Rat anti-mouse Ly6G (clone 1A8; BD Biosciences); rat antimouse F4/80 (clone: BM8), CD11b (clone: Mac-1) and CD41 (clone: eBioMWReg30) were from eBioscience. Human recombinant granulocyte-monocyte colony stimulating factor (GM-CSF) and LC grade solvents were purchased from Fisher Scientific; Agilent Eclipse C18 (4.6 mm $\times 100 \mathrm{~mm} \times 1.8 \mu \mathrm{m} ; 4.6 \mathrm{~mm} \times 50 \mathrm{~mm} \times$ $1.8 \mu \mathrm{m}$ ) column; C18 SPE columns (Biotage); fluorescently conjugated Zymosan A (Invitrogen); LC-MS-MS quantification and deuterated internal standards $\left(\mathrm{d}_{8}-5 S\right.$ hydroxyeicosatetraenoic acid $\left(\mathrm{d}_{8}-5 S\right.$-HETE), $\mathrm{d}_{4}-\mathrm{LTB}_{4}, \mathrm{~d}_{5}$-lipoxin $\mathrm{A}_{4}\left(\mathrm{~d}_{5}-\mathrm{LXA}_{4}\right)$, $\mathrm{d}_{4}-\mathrm{PGE}_{2}, \mathrm{RvD1}, \mathrm{RvD2}$; Cayman Chemicals).

Animals. All animals used in the present study were male FVB mice (Charles River Laboratories) that were 6-8 weeks old (weighing $20-25 \mathrm{~g}$ ). They were maintained in a temperature-and light-controlled environment and had unlimited access to water and food (laboratory standard rodent diet 5001 (Lab Diet)), containing 1.5\% eicosapentaenoic acid, 1.9\% DHA of total fatty acids. Experiments were performed in accordance with the Harvard Medical School Standing Committee on Animals guidelines for animal care (Protocol 02570).

n-3 DPA-derived mediator preparations. Exudates were obtained from mice $12 \mathrm{~h}$ after zymosan administration and incubated with n-3 DPA in DPBS $(1 \mu \mathrm{M}, 45 \mathrm{~min}$ $37^{\circ} \mathrm{C}, \mathrm{pH} 7.45$ ); the incubations were stopped using 2 volumes of ice-cold methanol and products extracted as outlined in the sample extraction and lipid mediator metabololipidomics section below.

$14 S$-HpDPA was prepared from n-3 DPA $(\sim 15 \mu \mathrm{M})$ incubated with $5.4 \mathrm{U} / \mathrm{ml}$ isolated 12-lipoxygenase (LOX) (porcine) $(0.05 \mathrm{M}$ phosphate buffer, $0.02 \%$ Tween 20, pH 7.4). 14S-HpDHA was isolated via RP-HPLC (1100 Series; Agilent

Technologies) using a C18 column and a mobile phase consisting of methanol/water $(60: 20, \mathrm{vol} / \mathrm{vol})$ at $0.5 \mathrm{ml} / \mathrm{min}$ that was ramped up to $98: 2 \mathrm{vol} / \mathrm{vol}$ over for $20 \mathrm{~min}$. Reduction with $\mathrm{NaBH}_{4}$ yielded 14S-HDPA used for mass spectrometry standard. In determined incubations $14 S$-HpDPA was incubated with human macrophages $(40 \times$ $\left.10^{6} / \mathrm{ml}\right)$ or neutrophils $\left(80 \times 10^{6} / \mathrm{ml}\right)$ in PBS (containing calcium and magnesium) and serum treated zymosan $(\mathrm{StZ}, 0.1 \mathrm{mg} / \mathrm{ml})$; the incubations were stopped after $45 \mathrm{~min}$ and products extracted.

17S-HpDPA was prepared from n-3 DPA $(\sim 15 \mu \mathrm{M})$ incubated with $100 \mathrm{U} / \mathrm{ml}$ isolated soybean-LOX (Borate buffer, $4^{\circ} \mathrm{C}, \mathrm{pH} 9.2$ ). 17S-HpDHA was isolated via RPHPLC. Reduction with $\mathrm{NaBH}_{4}$ yielded $17 \mathrm{~S}$-HDPA used for mass spectrometry standard. Biogenic synthesis of the di- and tri- dioxygenation products was performed with 5-LOX enzyme ( $200 \mathrm{U} / \mathrm{ml}$ ) incubated with $17 \mathrm{~S}$-HpDPA. In determined incubations, $17 \mathrm{Hp}$-DPA was added to human macrophages $\left(40 \times 10^{6} / \mathrm{ml}\right)$ or neutrophils $\left(80 \times 10^{6} / \mathrm{ml}\right)$ in PBS (containing calcium and magnesium) and cells were then incubated with StZ $(0.1 \mathrm{mg} / \mathrm{ml})$, incubations were stopped after $45 \mathrm{~min}$ and products extracted. These were scaled up for direct comparison of biological and physical properties with other novel compounds isolated from peripheral blood leukocytes or inflammatory exudates.

Ischemia reperfusion injury. Mice were anesthetized by intraperitoneal injection of a mixture of xylazine $(80 \mathrm{mg} / \mathrm{Kg})$ and ketamine $(10 \mathrm{mg} / \mathrm{Kg})$. Hind-limb ischemia was initiated using tourniquets consisting of a rubber band placed on each hind limb as in $^{9}$. Ten min prior to the initiation of reperfusion n-3 DPA products (obtained as described above) or Resolvin D1 ( $0.5 \mathrm{ng}$ ) were administered by intravenous injection and compared to vehicle alone. At the end of this reperfusion period $(2 \mathrm{~h})$, mice were euthanized and blood collected via cardiac puncture, lungs harvested, frozen in liquid nitrogen and stored at $-80^{\circ} \mathrm{C}$ or stored in $10 \%(\mathrm{v} / \mathrm{v})$ buffered formalin and processed for histology by the Children's Hospital Boston Core Histology Facility. The frozen lungs were homogenized from individual mice, centrifuged and the tissue myeloperoxidase (MPO) levels were determined using a mouse MPO ELISA (R\&D Systems.).

To investigate platelet leukocyte aggregates in murine whole blood after ischemia reperfusion, blood was collected $2 \mathrm{~h}$ post reperfusion by cardiac puncture and incubated with PE conjugated rat anti-mouse Ly6G and FITC conjugated rat antimouse $\mathrm{CD} 41$ or relevant isotype controls for $30 \mathrm{~min}$ at $4{ }^{\circ} \mathrm{C}$. Red blood cells were lysed 
using ice-cold red-blood cell lysis buffer (BD Biosciences) and cells fixed with $1 \%$ formalin prior to analysis by a BD Canto II. Data was analyzed using FlowJo (TreeStar Inc.).

Sample extraction and lipid mediator metabololipidomics. All samples for LC-MSMS analysis were extracted on SPE columns as in Ref. 41. Prior to extraction, 500 pg of deuterium-labeled internal standards $\mathrm{d}_{8}-5 S$-HETE, $\mathrm{d}_{4} \mathrm{LTB}_{4}, \mathrm{~d}_{5} \mathrm{LXA}_{4}$ and $\mathrm{d}_{4} \mathrm{PGE}_{2}$ were added to facilitate quantification of sample recovery.

The LC-MS-MS system, QTrap 5500 (ABSciex), was equipped with an Agilent HP1100 binary pump and diode-array detector (DAD). An Agilent Eclipse Plus C18 column $(100 \mathrm{~mm} \times 4.6 \mathrm{~mm} \times 1.8 \mu \mathrm{m})$ was used with a gradient of methanol/water/ acetic acid of $60: 40: 0.01(\mathrm{v} / \mathrm{v} / \mathrm{v})$ to $100: 0: 0.01$ at $0.4 \mathrm{ml} / \mathrm{min}$ flow rate. To monitor and quantify the levels of the various LM, a multiple reaction monitoring (MRM) method was developed with signature ion fragments for each molecule. Identification was conducted using published criteria ${ }^{17}$ with at least six diagnostic ions. Calibration curves were obtained using synthetic LM mixture $\left(\mathrm{d}_{8}-5 S\right.$-HETE, $\mathrm{d}_{4} \mathrm{LTB}_{4}, \mathrm{~d}_{5} \mathrm{LXA}_{4}$, $\mathrm{d}_{4} \mathrm{PGE}_{2}, \mathrm{TXB}_{2}, \mathrm{PGD}_{2}, \mathrm{PGF}_{2 \alpha}, \mathrm{RvD} 1, \mathrm{RvD} 2, \mathrm{RvD} 5$, Protectin (PD)1, Maresin 1 (MaR1), 17-hydroxydocosahexaenoic acid (17-HDHA), 14-hydroxydocosahexaenoic acid (14-HDHA) and 7-hydroxydocosahexaenoic acid (7-HDHA) at 1, 10, 100, $275 \mathrm{pg}$. Linear calibration curves for each were obtained with $\mathrm{r}^{2}$ values in the range 0.98-0.99. Quantification was carried out based on peak area of the Multiple Reaction Monitoring (MRM) transition and the linear calibration curve for each compound. Where calibration curves for a structurally related DHA-derived product were not available (14,21-diHDPA, 13,14-diHDPA and 16,17-diHDPA), levels were monitored using a compound with similar physical properties.

For chiral lipidomic analysis, a Chiralpak AD-RH column $(150 \mathrm{~mm} \times 2.1 \mathrm{~mm} \times$ $5 \mu \mathrm{m})$ was used with isocratic methanol/water/acetic acid $95: 5: 0.01(\mathrm{v} / \mathrm{v} / \mathrm{v})$ at $0.15 \mathrm{ml} / \mathrm{min}$. To monitor isobaric monohydroxy docosapentaenoic acid levels, a multiple reaction monitoring (MRM) method was developed using signature ion fragments for each molecule.

Zymosan peritonitis. Zymosan $(0.1 \mathrm{mg}$ ) was injected intraperitoneally (i.p.) in $1 \mathrm{ml}$ of sterile saline. Exudates were collected at $0,4,12$ and 24 h post zymosan. Leukocyte numbers and differential counts in the peritoneal exudates were determined as in ${ }^{16}$. In designated experiments, mice were administered intravenously (i.v): Vehicle (saline containing $0.1 \% \mathrm{EtOH}$ ), or the indicated mixture of n-3 DPA-derived products at $100 \mathrm{ng} /$ mouse $5 \mathrm{~min}$ prior to i.p. zymosan administration $(0.1 \mathrm{mg})$. After $4 \mathrm{~h}$ the exudates were collected and the number of extravasated neutrophils determined using Turks solution and flow cytometry as above.

Neutrophil isolation and chemotaxis. Peripheral blood neutrophils were obtained from healthy volunteers as in Ref. 17. Briefly, neutrophils were prepared following density separation by layering on Ficoll-Histopaque 1077-1. The cells were then centrifuged at $300 \mathrm{~g}\left(30 \mathrm{~min}, 4^{\circ} \mathrm{C}\right)$, and contaminating red blood cells were lysed by hypotonic lyses. These were then suspended at $1 \times 10^{6}$ cells $/ \mathrm{ml}$ in DPBS containing $0.1 \%$ BSA and incubated with vehicle $(0.1 \% \mathrm{EtOH}$ in DPBS), RvD2 ( $1 \mathrm{nM})$ or the indicated mixtures of n-3 DPA-derived products $(1 \mathrm{nM})$ for $15 \mathrm{~min}$ at $37^{\circ} \mathrm{C} .2 .5 \times$ $10^{4}$ cells were then added to the upper chamber of the ChemoTx System $(3 \mu \mathrm{m}$ pore size filter) and chemotaxis towards IL $8(100 \mathrm{ng} / \mathrm{ml})$ was assessed $\left(90 \mathrm{~min}, 37^{\circ} \mathrm{C}, 5 \%\right.$ $\mathrm{CO}_{2}$ ). The number of cells migrated into the bottom chamber was determined using Alama Blue following manufacturer's instructions on a Spectra Max M3 microplate reader (Molecular Devices Inc., Sunnyvale, CA).

Human neutrophil-endothelial cell adhesion. HUVEC were purchased from Lonza and cultured to passage 4. The cells were plated onto 96-well plates (Costar) coated with $1 \%$ gelatin and incubated overnight. The HUVEC were then incubated with TNF- $\alpha\left(10 \mathrm{ng} / \mathrm{ml}, 4 \mathrm{~h}, 37^{\circ} \mathrm{C}\right)$ in media containing $0.1 \%$ fetal bovine serum. Human peripheral blood neutrophils were isolated as described above and labeled with CFDA as in Ref. 17. These were then suspended in DPBS and incubated with vehicle $(0.1 \%$ EtOH) or the indicated mixture of n-3 DPA products $\left(1 \mathrm{nM}, 37^{\circ} \mathrm{C}, \mathrm{pH} 7.45\right)$. After 15 min the PMN $\left(1 \times 10^{5}\right)$ were added to the HUVEC and incubated for $60 \mathrm{~min}$ $\left(37^{\circ} \mathrm{C}\right)$. The plates were then washed with DPBS to remove non-adherent cells and the number of adherent neutrophils assessed using a Spectra Max M3 microplate reader.

Endothelial cell adhesion molecule expression. HUVEC were plated onto $1 \%$ gelatin coated 12-well plates and incubated overnight. Cells were then incubated with vehicle $(0.1 \% \mathrm{EtOH}$ in DPBS) or the indicated mixture of $n-3$ DPA products for $15 \mathrm{~min}$. The HUVEC were then incubated with TNF- $\alpha\left(10 \mathrm{ng} / \mathrm{ml}, 37^{\circ} \mathrm{C}\right)$ for $4 \mathrm{~h}$. At the end of these incubations, ICAM-1 levels were assessed by flow cytometry following staining with fluorescently conjugated mouse anti-human ICAM-1 antibody (Clone HCD54; BioLegend) using the staining protocol ${ }^{17}$.

Macrophage preparation and phagocytosis. Macrophages were prepared and phagocytosis was assessed as in Ref. 16. Briefly, cells were incubated with vehicle $(0.1 \% \mathrm{EtOH}$ in DPBS), RvD2 $(1 \mathrm{nM})$ or the indicated n-3 DPA products $(1 \mathrm{nM})$ for $15 \mathrm{~min}$ at $37^{\circ} \mathrm{C}$, then FITC-labeled zymosan was added and cells incubated $60 \mathrm{~min}$ at $37^{\circ} \mathrm{C}$. Phagocytosis was assessed using an M3 SpectraMax plate reader.

Statistics. All results are expressed as means \pm SEM. Differences between groups were compared using Student's t test (2 groups) or 1-way ANOVA (multiple groups) followed by post hoc Bonferroni test. The criterion for statistical significance was $P<0.05$.
1. Serhan, C. N. \& Savill, J. Resolution of inflammation: the beginning programs the end. Nat Immunol 6(12), 1191-1197 (2005).

2. Serhan, C. N. A search for endogenous mechanisms of anti-inflammation uncovers novel chemical mediators: missing links to resolution. Histochem Cell Biol 122(4), 305-321 (2004).

3. Tabas, I. \& Glass, C. K. Anti-inflammatory therapy in chronic disease: challenges and opportunities. Science 339(6116), 166-172 (2013).

4. Buckley, C. D., Gilroy, D. W., Serhan, C. N., Stockinger, B. \& Tak, P. P. The resolution of inflammation. Nat Rev Immunol 13(1), 59-66 (2013).

5. Samuelsson, B. Role of basic science in the development of new medicines: examples from the eicosanoid field. J Biol Chem 287(13), 10070-10080 (2012).

6. Shimizu, T. Lipid mediators in health and disease: enzymes and receptors as therapeutic targets for the regulation of immunity and inflammation. Annu Rev Pharmacol Toxicol 49, 123-150 (2009).

7. Chiang, N., Fredman, G., Backhed, F., Oh, S. F., Vickery, T. \& Schmidt, B. A. et al Infection regulates pro-resolving mediators that lower antibiotic requirements. Nature 484(7395), 524-528 (2012).

8. Flower, R. J. \& Vane, J. R. Inhibition of prostaglandin biosynthesis. Biochem Pharmacol 23(10), 1439-1450 (1974).

9. Kasuga, K., Yang, R., Porter, T. F., Agrawal, N., Petasis, N. A. \& Irimia, D. et al. Rapid appearance of resolvin precursors in inflammatory exudates: novel mechanisms in resolution. J Immunol 181(12), 8677-8687 (2008).

10. Calder, P. C. Fatty acids and inflammation: the cutting edge between food and pharma. Eur J Pharmacol 668 Suppl 1, S50-58 (2011).

11. Rapoport, S. I. Translational studies on regulation of brain docosahexaenoic acid (DHA) metabolism in vivo. Prostaglandins Leukot Essent Fatty Acids 88(1), 79-85 (2013).

12. De Caterina, R. n-3 fatty acids in cardiovascular disease. N Engl J Med 364(25), 2439-2450 (2011).

13. Colgan, S. P., Ehrentraut, S. F., Glover, L. E., Kominsky, D. J. \& Campbell, E. L. Contributions of neutrophils to resolution of mucosal inflammation. Immunol Res 55(1-3), 75-82 (2013).

14. Serhan, C. N., Clish, C. B., Brannon, J., Colgan, S. P., Chiang, N. \& Gronert, K. Novel functional sets of lipid-derived mediators with antiinflammatory actions generated from omega-3 fatty acids via cyclooxygenase 2-nonsteroidal antiinflammatory drugs and transcellular processing. J Exp Med 192(8), 1197-1204 (2000).

15. Serhan, C. N., Hong, S., Gronert, K., Colgan, S. P., Devchand, P. R. \& Mirick, G. et al. Resolvins: a family of bioactive products of omega- 3 fatty acid transformation circuits initiated by aspirin treatment that counter proinflammation signals. J Exp Med 196(8), 1025-1037 (2002).

16. Serhan, C. N., Dalli, J., Karamnov, S., Choi, A., Park, C. K. \& Xu, Z. Z. et al Macrophage proresolving mediator maresin 1 stimulates tissue regeneration and controls pain. FASEB J 26(4), 1755-1765 (2012).

17. Dalli, J. \& Serhan, C. N. Specific lipid mediator signatures of human phagocytes: microparticles stimulate macrophage efferocytosis and pro-resolving mediators. Blood 120(15), e60-72 (2012).

18. Zhang, M. J. \& Spite, M. Resolvins: anti-inflammatory and proresolving mediators derived from omega-3 polyunsaturated fatty acids. Annu Rev Nutr 32, 203-227 (2012).

19. Giera, M., Ioan-Facsinay, A., Toes, R., Gao, F., Dalli, J. \& Deelder, A. M. et al. Lipid and lipid mediator profiling of human synovial fluid in rheumatoid arthritis patients by means of LC-MS/MS. Biochim Biophys Acta 1821(11), 1415-1424 (2012).

20. Mas, E., Croft, K. D., Zahra, P., Barden, A. \& Mori, T. A. Resolvins D1, D2, and Other Mediators of Self-Limited Resolution of Inflammation in Human Blood following n-3 Fatty Acid Supplementation. Clin Chem 58(10), 1476-1484 (2012)

21. Lemaitre, R. N., Tanaka, T., Tang, W., Manichaikul, A., Foy, M. \& Kabagambe, E. K. et al. Genetic loci associated with plasma phospholipid n-3 fatty acids: a meta-analysis of genome-wide association studies from the CHARGE Consortium. PLoS Genet 7(7), e1002193 (2011).

22. Crawford, M. A., Leigh Broadhurst, C., Guest, M., Nagar, A., Wang, Y. \& Ghebremeskel, K. et al. A quantum theory for the irreplaceable role of docosahexaenoic acid in neural cell signalling throughout evolution. Prostaglandins Leukot Essent Fatty Acids 88(1), 5-13 (2013).

23. Hussein, N., Fedorova, I., Moriguchi, T., Hamazaki, K., Kim, H. Y. \& Hoshiba, J. et al. Artificial rearing of infant mice leads to n-3 fatty acid deficiency in cardiac, neural and peripheral tissues. Lipids 44(8), 685-702 (2009).

24. van Gils, J. M., Zwaginga, J. J. \& Hordijk, P. L. Molecular and functional interactions among monocytes, platelets, and endothelial cells and their relevance for cardiovascular diseases. J Leukoc Biol 85(2), 195-204 (2009).

25. Bannenberg, G. L., Chiang, N., Ariel, A., Arita, M., Tjonahen, E. \& Gotlinger, K. H. et al. Molecular circuits of resolution: formation and actions of resolvins and protectins. J Immunol 174(7), 4345-4355 (2005).

26. Granger, D. N. \& Kubes, P. The microcirculation and inflammation: modulation of leukocyte-endothelial cell adhesion. J Leukoc Biol 55(5), $662-675$ (1994).

27. Spite, M., Norling, L. V., Summers, L., Yang, R., Cooper, D. \& Petasis, N. A. et al. Resolvin D2 is a potent regulator of leukocytes and controls microbial sepsis. Nature 461(7268), 1287-1291 (2009).

28. Luscinskas, F. W., Cybulsky, M. I., Kiely, J. M., Peckins, C. S., Davis, V. M. \& Gimbrone, M. A. Jr. Cytokine-activated human endothelial monolayers support enhanced neutrophil transmigration via a mechanism involving both endothelial- 
leukocyte adhesion molecule-1 and intercellular adhesion molecule-1. J Immunol 146(5), 1617-1625 (1991).

29. Sun, Q., Ma, J., Campos, H., Rexrode, K. M., Albert, C. M. \& Mozaffarian, D. et al. Blood concentrations of individual long-chain $\mathrm{n}-3$ fatty acids and risk of nonfatal myocardial infarction. Am J Clin Nutr 88(1), 216-223 (2008).

30. Khaw, K. T., Friesen, M. D., Riboli, E., Luben, R. \& Wareham, N. Plasma phospholipid fatty acid concentration and incident coronary heart disease in men and women: the EPIC-Norfolk prospective study. PLoS Med 9(7), e1001255 (2012).

31. Oda, E., Hatada, K., Katoh, K., Kodama, M., Nakamura, Y. \& Aizawa, Y. A casecontrol pilot study on $\mathrm{n}-3$ polyunsaturated fatty acid as a negative risk factor for myocardial infarction. Int Heart J 46(4), 583-591 (2005).

32. Rissanen, T., Voutilainen, S., Nyyssonen, K., Lakka, T. A. \& Salonen, J. T. Fish oilderived fatty acids, docosahexaenoic acid and docosapentaenoic acid, and the risk of acute coronary events: the Kuopio ischaemic heart disease risk factor study. Circulation 102(22), 2677-2679 (2000)

33. Qiu, F. H., Wada, K., Stahl, G. L. \& Serhan, C. N. IMP and AMP deaminase in reperfusion injury down-regulates neutrophil recruitment. Proc Natl Acad Sci U S A 97(8), 4267-4272 (2000).

34. Serhan, C. N. \& Petasis, N. A. Resolvins and protectins in inflammation resolution. Chem Rev 111(10), 5922-5943 (2011).

35. Serhan, C. N., Yang, R., Martinod, K., Kasuga, K., Pillai, P. S. \& Porter, T. F. et al. Maresins: novel macrophage mediators with potent antiinflammatory and proresolving actions. J Exp Med 206(1), 15-23 (2009).

36. Furman, M. I., Dauerman, H. L., Goldberg, R. J., Yarzebski, J., Lessard, D. \& Gore, J. M. Twenty-two year (1975 to 1997) trends in the incidence, in-hospital and long-term case fatality rates from initial Q-wave and non-Q-wave myocardial infarction: a multi-hospital, community-wide perspective. J Am Coll Cardiol 37(6), 1571-1580 (2001).

37. Spite, M. \& Serhan, C. N. Novel lipid mediators promote resolution of acute inflammation: impact of aspirin and statins. Circ Res 107(10), 1170-1184 (2010).

38. Dangi, B., Obeng, M., Nauroth, J. M., Chung, G., Bailey-Hall, E. \& Hallenbeck, T. et al. Metabolism and biological production of resolvins derived from docosapentaenoic acid (DPAn-6). Biochem Pharmacol 79(2), 251-260 (2010).

39. Chiu, C. Y., Gomolka, B., Dierkes, C., Huang, N. R., Schroeder, M. \& Purschke, M. et al. Omega-6 docosapentaenoic acid-derived resolvins and 17 hydroxydocosahexaenoic acid modulate macrophage function and alleviate experimental colitis. Inflamm Res 61(9), 967-976 (2012).

40. Barrett, E., Fitzgerald, P., Dinan, T. G., Cryan, J. F., Ross, R. P. \& Quigley, E. M. et al. Bifidobacterium breve with alpha-Linolenic Acid and Linoleic Acid Alters Fatty Acid Metabolism in the Maternal Separation Model of Irritable Bowel Syndrome. PLoS One 7(11), e48159 (2012).

41. Yang, R., Chiang, N., Oh, S. F. \& Serhan, C. N. Metabolomics-lipidomics of eicosanoids and docosanoids generated by phagocytes. Curr Protoc Immunol Chapter 14, Unit 1426 (2011).

\section{Acknowledgements}

The authors thank Mary Halm Small for expert assistance in manuscript preparation. This work was supported in part by the National Institutes of Health (grant P01GM095467).

\section{Author contributions}

J.D. designed and carried out experiments, analyzed data, and contributed to manuscript and figure preparations; R.A.C. carried out experiments, analyzed data, and contributed to manuscript and figure preparations; C.N.S. carried out overall experimental design, conceived the research plan, and contributed to manuscript and figure preparations.

\section{Additional information}

Supplementary information accompanies this paper at http://www.nature.com/ scientificreports

Competing financial interests: A patent application is submitted assigned to Brigham and Women's Hospital with C.N.S. and J.D. as inventors.

License: This work is licensed under a Creative Commons

Attribution-NonCommercial-NoDerivs 3.0 Unported License. To view a copy of this license, visit http://creativecommons.org/licenses/by-nc-nd/3.0/

How to cite this article: Dalli, J., Colas, R.A. \& Serhan, C.N. Novel n-3 Immunoresolvents Structures and Actions. Sci. Rep. 3, 1940; DOI:10.1038/srep01940 (2013). 


\section{SCIENTIFIC \\ REP RTS}

DOI: $10.1038 /$ srep06726

SUBJECT AREAS: CORRIGENDUM: Novel n-3 Immunoresolvents: Structures and Actions

EXPERIMENTAL MODELS

OF DISEASE

ACUTE INFLAMMATION

INNATE IMMUNITY

CHRONIC INFLAMMATION

\section{SCIENTIFIC REPORTS:}

$3: 1940$

DOI: $10.1038 /$ srep01940

(2013)

Published:

5 June 2013

Updated:

24 October 2014

\section{Jesmond Dalli, Romain A. Colas \& Charles N. Serhan}

This Article contains an error in the chemical structure for RvD2 $2_{n-3}$ DPA in both Figure 8 a and Supplementary Figure $3 \mathrm{~b}$ where a carbon is missing from the omega end of the molecule. The correct Figure $8 \mathrm{a}$ and Supplementary Figure $3 \mathrm{~b}$ appear below as Figure 1 and Figure 2 respectively.

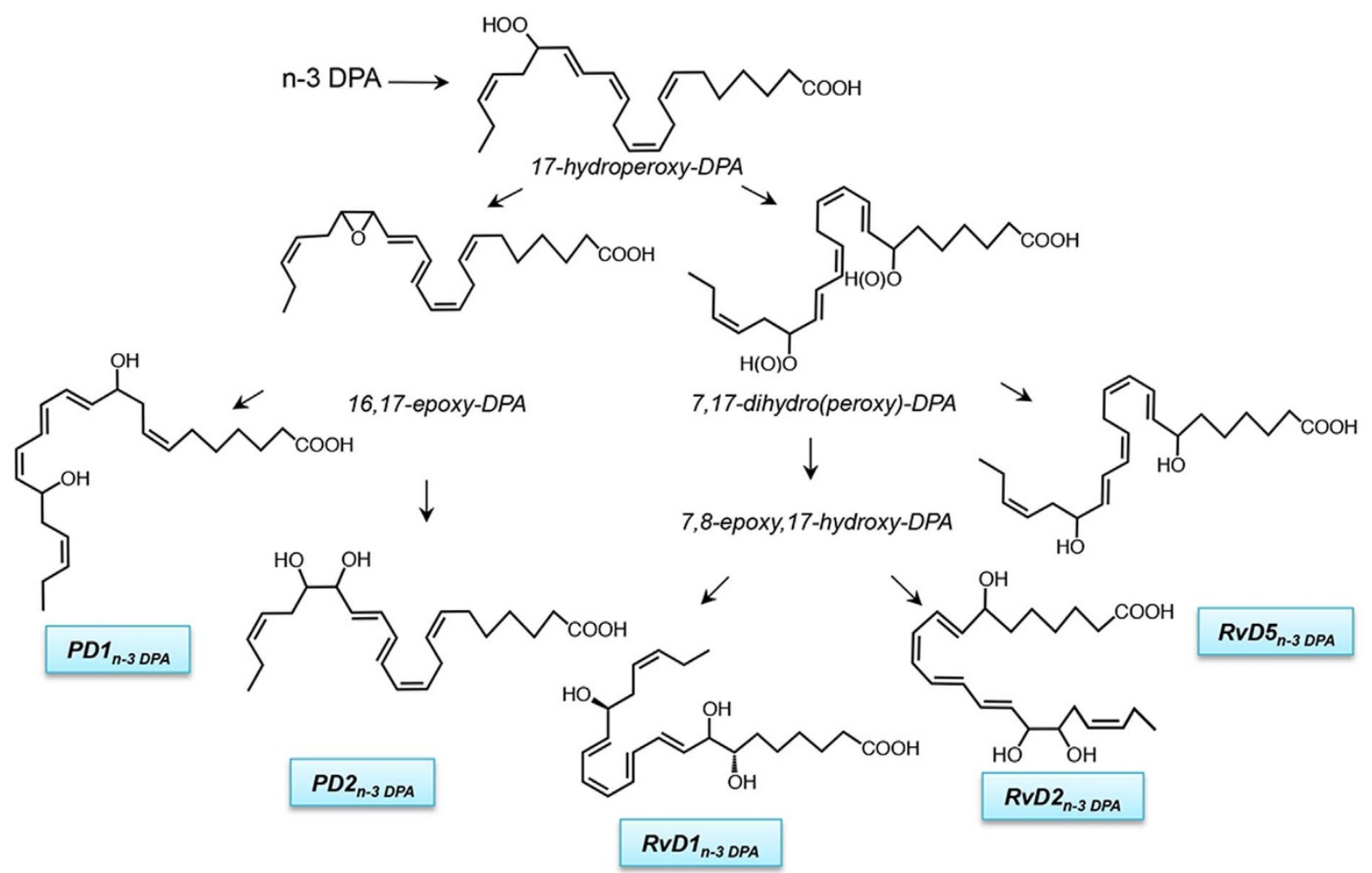

Figure 1 | 


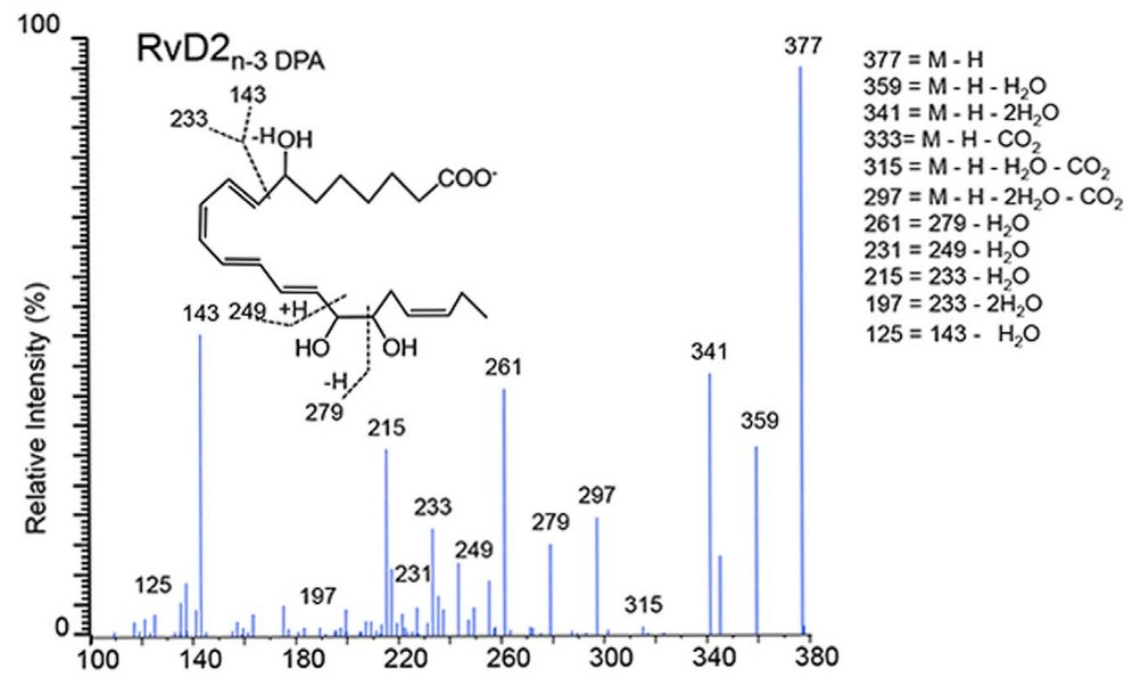

Figure 2 